\title{
Topological Quantum Field Theories from Compact Lie Groups
}

\section{Citation}

Freed, Daniel, Michael J. Hopkins, Jacob Alexander Lurie, and Constantin Teleman. 2010.

Topological quantum field theories from compact lie groups. In A Celebration of the Mathematical Legacy of Raoul Bott, vol. 50 of CRM Proceedings and Lecture Notes, ed. P. Robert Kotiuga, 367-403. Providence, RI: American Mathematical Society.

\section{Permanent link}

http://nrs.harvard.edu/urn-3:HUL.InstRepos:10009465

\section{Terms of Use}

This article was downloaded from Harvard University's DASH repository, and is made available under the terms and conditions applicable to Open Access Policy Articles, as set forth at http:// nrs.harvard.edu/urn-3:HUL.InstRepos:dash.current.terms-of-use\#OAP

\section{Share Your Story}

The Harvard community has made this article openly available.

Please share how this access benefits you. Submit a story.

Accessibility 


\title{
TOPOLOGICAL QUANTUM FIELD THEORIES FROM COMPACT LIE GROUPS
}

\author{
DANIEL S. FREED, MICHAEL J. HOPKINS, JACOB LURIE, AND CONSTANTIN TELEMAN
}

Let $G$ be a compact Lie group and $B G$ a classifying space for $G$. Then a class in $H^{n+1}(B G ; \mathbb{Z})$ leads to an $n$-dimensional topological quantum field theory (TQFT), at least for $n=1,2,3$. The theory for $n=1$ is trivial, but we include it for completeness. The theory for $n=2$ has some infinities if $G$ is not a finite group; it is a topological limit of 2-dimensional Yang-Mills theory. The most direct analog for $n=3$ is an $L^{2}$ version of the topological quantum field theory based on the classical Chern-Simons invariant, which is only partially defined. The TQFT constructed by Witten and Reshetikhin-Turaev which goes by the name 'Chern-Simons theory' (sometimes 'holomorphic Chern-Simons theory' to distinguish it from the $L^{2}$ theory) is completely finite.

The theories we construct here are extended, or multi-tiered, TQFTs which go all the way down to points. For the $n=3$ Chern-Simons theory, which we term a '0-1-2-3 theory' to emphasize the extension down to points, we only treat the cases where $G$ is finite or $G$ is a torus, the latter being one of the main novelties in this paper. In other words, for toral theories we provide an answer to the longstanding question: What does Chern-Simons theory attach to a point? The answer is a bit subtle as Chern-Simons is an anomalous field theory of oriented manifolds 1 This framing anomaly was already flagged in Witten's seminal paper [Wi]. Here we interpret the anomaly as an invertible 4-dimensional topological field theory $\mathscr{A}$, defined on oriented manifolds. The Chern-Simons theory is a "truncated morphism" $Z: 1 \rightarrow \mathscr{A}$ from the trivial theory to the anomaly theory. For example, on a closed oriented 3-manifold $X$ the anomaly theory produces a complex line $\mathscr{A}(X)$ and the Chern-Simons invariant $Z(X)$ is a (possibly zero) element of that line. This is the standard vision of an anomalous quantum field theory in general; here we use this description down to points. The invariant of a 4 -manifold in the theory $\mathscr{A}$ involves its signature and Euler characteristic. It was first discovered in a combinatorial description [CY] and Walker [W] also uses $\mathscr{A}$ in his description of Chern-Simons (for a more general class of gauge groups).

Since a torus is an abelian group, the classical Chern-Simons action is quadratic in the connection and so the theory is in some sense "free". Indeed, one expects that the semi-classical approximation is exact. This is the point of view taken by Manoliu [Ma], who constructs Chern-Simons for circle groups as a 2-3 theory. The invariant this theory assigns to a closed oriented 3-manifold is made from classical invariants of 3-manifold topology: is the integral over the space of flat connections of the square root of the Reidemeister torsion times a spectral flow phase. (There is an overall

Date: June 19, 2009.

The work of D.S.F. is supported by NSF grant DMS-0603964.

The work of M.J.H. is supported by NSF grants DMS-0306519 and DMS-0757293 as well as the DARPA grant FA9550-07-1-0555.

The work of J.L. is supported by the American Institute of Mathematics.

The work of C.T. is supported by NSF grant DMS-0709448.

${ }^{1}$ It is not anomalous as a theory of framed manifolds. 
"volume" factor as well.) The extension to a 1-2-3 theory is determined by a modular tensor category by a theorem of Reshetikhin and Turaev [T]; it is assigned to the circle by the theory. For toral Chern-Simons this is a well-known category, and its relation to Chern-Simons theory was explored recently by Stirling [St], inspired by earlier work by Belov-Moore [BM]. In a different direction the Verlinde ring, which encodes the 2-dimensional reduction of Chern-Simons theory, was recognized by three of the authors as a twisted form of equivariant $K$-cohomology [FHT]. That description, or rather its equivalent in $K$-homology, inspires the categories of skyscraper sheaves which we use to extend toral Chern-Simons to a 0-1-2-3 theory.

The gauge theories discussed in this paper have a classical description as pure gauge theories whose only field is a $G$-bundle with connection. On any fixed manifold $M$ the $G$-bundles with connection form a groupoid, and it is the underlying stack which should be regarded as the fields in the theory. When $M$ is a point, there is up to isomorphism a unique $G$-bundle with connection (the trivial one), but it has a group of automorphisms isomorphic to $G$. Thus the stack of $G$-bundles with connections is $* / / G=B G$. A cohomology class in $H^{n+1}(B G ; \mathbb{Z})$, or rather the geometric manifestation which we describe (esp. §4.1 and §5.1), defines the classical gauge theory on $M \simeq$ pt. We directly "quantize" this classical data over a point. This may be regarded, as in [F2], as the "path integral" over the fields on a point. Integration over $I / / G$, which is implemented as a categorical limit, amounts to taking $G$-invariants, which is a higher version of the Gauss Law in canonical quantization. It is also worth noting that the space of connections on $M=S^{1}$ is the finite dimensional groupoid $G / / G$, where $G$ acts on $G$ by conjugation. Properly interpreted, this is the loop space of the fields on a point. On the other hand, for any manifold of dimension at least two, the stack of connections is infinite dimensional unless $G$ is finite.

In the latter case - for a finite group $G$ - the path integral in all dimensions reduces to a finite sum, so is manifestly well-defined and satisfies the axioms of a field theory. This idea was initiated in [F2], where the notion of a classical action in an $n$-dimensional field theory is extended to manifolds of all dimensions $<n$ and a higher categorical version of the path integral is used to heuristically define the quantum invariants.2 The developments in higher category theory in the intervening years, and the new definitions and structure theory for TQFT, make it possible to give a rigorous treatment of these finite path integrals and to generalize them. We give some indications in $₫ 3$ and 98 , we hope to develop these ideas in detail elsewhere.

That structure theorem is the Baez-Dolan cobordism hypothesis, for which we include an exposition in $\$ 2.3$. A much more detailed account may be found in $[\mathrm{L}$, which also contains a detailed sketch of the proof. This theorem asserts that a fully extended TQFT - that is, a TQFT extended down to points - is determined by its value on a point. Furthermore, it characterizes the possible values on a point. This theorem provides one possible construction of many of the theories in this paper 3

The anomaly theory $\mathscr{A}$ which appears in Chern-Simons theory has a classical description as a 4-dimensional field theory. In $\$ 5.2$ we sketch two such classical theories, each based on a 2-groupoid

\footnotetext{
${ }^{2}$ The application in $\mathrm{F} 2$ is to derive the quantum group which appears in 3-dimensional Chern-Simons theory from the classical Chern-Simons action.

${ }^{3}$ A corollary of the cobordism hypothesis characterizes theories on oriented and spin manifolds: for these the value on a point is endowed with certain equivariance data. For the 3- and 4-dimensional theories of most interest we do not pursue this equivariance data here.
} 
of gerbes (rather than a 1-groupoid of connections, which is what appears in gauge theory). These are free theories, so the path integral on a 4-manifold is Gaussian and can be carried out explicitly. Presumably this is a classical description of the theories put forward by Crane and Yetter [CY], though we do not attempt to make the connection. One of these classical theories is based on a finite group, and for that we can apply the finite path integrals of 93 (in the generalized form for 2-groupoids) to construct the quantum theory. In 6 we start at the other end of the theory, that is, with points rather than 4-manifolds. We describe two braided tensor categories which may be attached to a point in the anomaly theory $\mathscr{A}$; they are Morita equivalent, so both define $\mathscr{A}$. $\operatorname{In} \S 6.2$ we introduce a finite (lattice) approximation of the continuous version of the anomaly theory, and so obtain many more Morita equivalent braided tensor categories which define that theory (Proposition 6.5). Finally, in $\S 9.1$ we spell out what the anomaly theory assigns to manifolds of various dimensions from 0 to 4 .

The Chern-Simons theory proper is described in $\S 9.2$, see Theorem 9.3 . The tables in $\S 9.3$ are helpful in organizing the motley characters who play a part in our story. Our account here emphasizes 0-manifolds as that is the new element.

We begin in $\$ 1$ with a discussion of the 1-dimensional case to set down some basic notions. Here the gauge group is an arbitrary compact Lie group $G$, and the path integral reduces to an integral over $G$. The 2-dimensional case (\$2) exhibits more features. In $\$$ we discuss the 3-dimensional gauge theory with finite gauge group. On the one hand this is an application of the finite path integral and on the other a warm-up for the treatment in later sections of torus groups. Section 7 is a set of variations on 'algebra' of increasing (categorical) complexity. It provides some underpinnings for our discussion of the anomaly theory $\mathscr{A}$ and Chern-Simons theory.

As befits a conference proceedings, our presentation here emphasizes the big picture and our speculation is uninhibited; many details are not worked out.

The recent paper [BN] generalizes the 3-dimensional untwisted Chern-Simons theory for finite groups in a different direction and gives applications to representation theory. Also, Bartels, Douglas, and Henriques have recently announced a construction of Chern-Simons theory for 1-connected compact Lie groups [D] which uses conformal nets and the cobordism hypothesis.

Raoul Bott was an inspiration to each of us, both personally and through his mathematics. We offer this paper as a tribute.

\section{The cohomology group $H^{2}(B G ; \mathbb{Z})$ and one-dimensional theories}

To begin, we recall the definition of a topological quantum field theory. Let $\left(\operatorname{Bord}_{n}^{S O}, \amalg\right)$ be the bordism category whose morphisms are oriented $n$-manifolds; it carries a symmetric monoidal structure given by disjoint union. Let $\left(\operatorname{Vect}_{\mathbb{C}}, \otimes\right)$ denote the symmetric monoidal category of finite dimensional complex vector spaces under tensor product.

Definition 1.1. An n-dimensional topological quantum field theory $F$ on oriented manifolds is a symmetric monoidal functor

$$
F:\left(\operatorname{Bord}_{n}^{S O}, \amalg\right) \longrightarrow\left(\operatorname{Vect}_{\mathbb{C}}, \otimes\right) .
$$


Thus a field theory assigns to each closed oriented $(n-1)$-manifold $Y$ a complex vector space $F(Y)$ and to each closed oriented $n$-manifold $X$ a number $F(X) \in \mathbb{C}$. If $X: Y_{0} \rightarrow Y_{1}$ is a compact manifold with boundary $-Y_{0} \amalg Y_{1}$, then $F(X): F\left(Y_{0}\right) \rightarrow F\left(Y_{1}\right)$ is a linear map. The functor $F$ maps the gluing operation of bordisms to the composition of linear maps. We refer the reader to $[\mathrm{A}], \mathrm{S}], \mathrm{L}]$ for more details and exposition about this definition.

There are many possible variations on Definition 1.1, For the domain we may take a bordism category of manifolds with other topological structures: oriented manifolds, spin manifolds, framed manifolds, etc. There are also other choices for the codomain. This definition describes "two-tiered theories"; we will see more tiers in 92 . If, say, $n=3$, then we designate this a ' $2-3$ theory' to emphasize the two tiers.

Let $n=1$. Then a theory $F$ assigns a vector space $F\left(\mathrm{pt}_{+}\right)$to the positively oriented point. We use the oriented interval with all possibilities for decomposing the boundary into incoming and outgoing components to deduce that $F\left(\mathrm{pt}_{-}\right)$is the dual space of $F\left(\mathrm{pt}_{+}\right)$and the intervals give the duality pairing. Finally, $F\left(S^{1}\right)=\operatorname{dim} F(\mathrm{pt})$, and the entire theory is determined up to isomorphism by this nonnegative integer.

We now describe 1-dimensional pure gauge theory with compact gauge group $G$ and action given by a class in $H^{2}(B G ; \mathbb{Z})$. Let $\mathbb{T} \subset \mathbb{C}$ be the circle group.

Proposition 1.2. There is an canonical isomorphism $\operatorname{Hom}(G, \mathbb{T}) \stackrel{\cong}{\rightrightarrows} H^{2}(B G ; \mathbb{Z})$.

In purely topological terms the classifying space $B G$ carries a universal principal $G$-bundle, and the isomorphism assigns to an abelian character $G \rightarrow \mathbb{T}$ the Chern class of the associated principal T-bundle. A more rigid viewpoint: abelian characters are in 1:1 correspondence with isomorphism classes of principal $\mathbb{T}$-bundle on the groupoid $* / / G$. A proof that the latter are classified by $H^{2}(B G ; \mathbb{Z})$ may be found in several texts, e.g. [AS, Proposition 6.3], Bry, Appendix].

Let $\lambda: G \rightarrow \mathbb{T}$ be an abelian character. Then the classical 1-dimensional gauge theory associated to $\lambda$ assigns to each $G$-bundle with connection on the circle the complex number $\lambda(g)$, where $g$ is the holonomy. This is what physicists term the 'exponentiated action' of the theory. Notice that holonomy depends on an orientation on the circle, so this is a theory of oriented 0 - and 1-manifolds. To the unique connection on a point is attached the trivial complex line $\mathbb{C}$; the automorphism group $G$ of the unique connection acts on $\mathbb{C}$ via $\lambda$. More precisely, this is correct if the point is positively oriented; for the negatively oriented point the action is via $\lambda^{-1}$. Now the standard quantization procedure constructs a theory $F$ with $F\left(\mathrm{pt}_{+}\right)$the elements in $\mathbb{C}$ invariant under the action of the automorphisms. (This is called the 'Gauss law' in physics.) The result is the zero vector space if $\lambda \neq 1$ is nontrivial and is $\mathbb{C}$ if $\lambda=1$ is trivial. Then $F\left(S^{1}\right)=0$ in the first case and $F\left(S^{1}\right)=1$ in the second, as $F\left(S^{1}\right)=\operatorname{dim} F\left(\mathrm{pt}_{+}\right)$. These values may be understood as the result of the path integral over the groupoid $G / / G$ of connections on $S^{1}$ with respect to Haar measure:

$$
\frac{1}{\operatorname{vol} G} \int_{G} \lambda(g) d g= \begin{cases}0, & \lambda \neq 1 \\ 1, & \lambda=1\end{cases}
$$

Remark 1.3. There are cohomology theories $h$ with the property that $h^{2}(B G)$ is isomorphic to the set of $\mathbb{Z} / 2 \mathbb{Z}$-graded abelian characters $(\lambda, \epsilon)$, i.e., $\lambda: G \rightarrow \mathbb{T}$ is an abelian character and $\epsilon=0,1$.

\footnotetext{
${ }^{4}$ Here ' $-Y_{0}$ ' denotes the oppositely oriented manifold.
} 
For example, we can take cohomology theory $h$ determined by a spectrum with two nontrivial homotopy groups: $\mathbb{Z}$ in degree 0 and $\mathbb{Z} / 2 \mathbb{Z}$ in degree -2 connected by the nontrivial $k$-invariant. A less efficient alternative is the Anderson dual of the sphere [HS, Definition B.2]. In either case spin structures are required to define pushforward, so the corresponding field theory is defined on spin manifolds. It is then natural to let the values of the theory lie in $\mathbb{Z} / 2 \mathbb{Z}$-graded complex vector spaces. If $\epsilon=0$, the theory factors through oriented manifolds and reduces to the previous. If $\epsilon=1$, then the classical theory assigns the odd line $\mathbb{C}$ to the unique connection on a point. For $\lambda \neq 1$ we obtain the trivial theory as above, but now if $\lambda=1$, then $F\left(S_{\mathrm{n}}^{1}\right)=-1$ and $F\left(S_{\mathrm{b}}^{1}\right)=1$, where the subscript indicates whether we consider the circle as endowed with the nonbounding (n) or bounding (b) spin structure.

There are similar generalizations of the higher dimensional theories discussed below.

Remark 1.4. All of the classical theories alluded to in this paper are purely topological and are most systematically defined by refining the class in $h^{n+1}(B G)$ to an object in the corresponding differential theory and then transgressing; see [F1] for the case $n=3$. The quantum theories, which are our focus, do not depend on the differential refinement.

\section{The cohomology group $H^{3}(B G)$ and two-dimensional theories}

\section{$\S 2.1 . H^{3}(B G ; \mathbb{Z})$ for arbitrary $G$}

Analogous to Proposition 1.2 we have the following.

Proposition 2.1. Let $G$ be a compact Lie group. The cohomology group $H^{3}(B G ; \mathbb{Z})$ can be identified with the set of isomorphism classes of central extensions $\mathbb{T} \rightarrow G^{\tau} \rightarrow G$.

In purely topological terms the class in $H^{3}(B G ; \mathbb{Z})$ attached to a central extension $\mathbb{T} \rightarrow G^{\tau} \rightarrow G$ is the obstruction to lifting the universal $G$-bundle to a $G^{\tau}$-bundle 5 A central extension is equivalently a smooth hermitian line bundle $K=K^{\tau} \rightarrow G$ together with isomorphisms

$$
\theta_{x, y}: K_{x} \otimes K_{y} \rightarrow K_{x y}, \quad x, y \in G
$$

for each pair in $G$ which satisfy an associativity constraint

$$
\theta_{x y, z}\left(\theta_{x, y} \otimes \operatorname{id}_{K_{z}}\right)=\theta_{x, y z}\left(\operatorname{id}_{K_{x}} \otimes \theta_{y, z}\right), \quad x, y, z \in G,
$$

for each triple in $G$. Recall the simplicial model

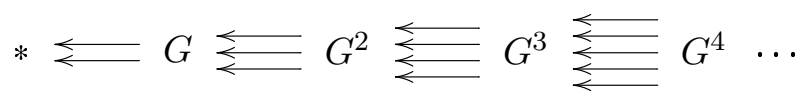

for $B G$, which is quite useful for computing cohomology $[\mathrm{B}]$. Write $S_{p}=G^{p}$ for the $p^{\text {th }}$ space in (2.4). In these terms a central extension is given by a line bundle $K^{\tau} \rightarrow S_{1}$, a trivialization (2.2)

\footnotetext{
${ }^{5}$ One familiar case is $\mathbb{T} \rightarrow \operatorname{Spin}_{n}^{c} \rightarrow S O_{n}$ in which case the obstruction is the third Stiefel-Whitney class $W_{3}$.
} 
of the alternating tensor product of its pullback to $S_{2}$, the latter constrained so that the alternating product of its pullbacks to $S_{3}$ is trivial (2.3). A proof of Proposition 2.1 may be found in AS, Proposition 6.3], [FHT, Part 1,§2.2.1], Bry, Appendix].

Recall that the complex group algebra $\mathbb{C}[G]$ of $G$ is the abelian group of complex functions on $G$ with multiplication given by convolution with respect to Haar measure, that is, pushforward under multiplication $m: G \times G \rightarrow G$. If $\operatorname{dim} G>0$ then we take the continuous functions on $G$, which form a topological ring using the sup norm. If $\operatorname{dim} G=0$ ( $G$ is finite) then the center of $\mathbb{C}[G]$ is the commutative algebra with natural basis the $\delta$-functions on the conjugacy classes. Alternatively, it is the subalgebra of central functions, a description which persists for all $G$.

Associated to a central extension $\mathbb{T} \rightarrow G^{\tau} \rightarrow G$ is a twisted complex group algebra $\mathbb{C}^{\tau}[G]$, the algebra of sections of $K^{\tau} \rightarrow G$ under convolution. Its center is again the commutative algebra of central functions.

\section{$\S 2.2$. Gauge theory with finite gauge group}

In an $n$-dimensional gauge theory with finite gauge group $G$ the path integral over a closed $n$-manifold $X$ reduces to a finite sum, and this sum defines the quantum invariant $F(X)$. The starting point of [F2] is an expansion of this standard picture: the classical action can be extended to manifolds of dimension $<n$ and a higher categorical version of the path integral determines the quantum invariants. For any manifold $M$ of dimension $\leqslant n$, the groupoid of $G$-bundles may be identified with the fundamental groupoid of the mapping space $\operatorname{Map}(M, B G)$. One can obtain a larger class of theories by replacing $B G$ with a topological space which has a finite number of nonzero homotopy groups, each of which is a finite group Q. At least heuristically, the path integrals on manifolds of dimension $\leqslant n$ define an extended field theory down to points which is manifestly local and functorial. We write these finite sums explicitly in this section. In §3 we indicate one possible path which should put these ideas on a rigorous footing.

For the 2-dimensional finite gauge theory based on the central extension $\mathbb{T} \rightarrow G^{\tau} \rightarrow G$ the classical invariant attached to a $G$-bundle $P \rightarrow X$ over a closed oriented 2-manifold is

$$
I(X, P)=e^{2 \pi i \sigma_{X}(P)}
$$

where $\sigma \in H^{2}(B G ; \mathbb{Q} / \mathbb{Z}) \cong H^{3}(B G ; \mathbb{Z})$ is the characteristic class corresponding to the central extension (see Proposition 2.1) and $\sigma_{X}(P) \in \mathbb{Q} / \mathbb{Z}$ is the characteristic number of the bundle. Physicists call $I(X, P)$ the 'exponentiated action' of the field $P$ on $X$. The quantum invariant of $X$ is then

$$
F(X)=\sum_{P} \frac{1}{\# \operatorname{Aut} P} I(X, P),
$$

where the sum is over a set of representative $G$-bundles on $X$, one in each equivalence class. For the trivial central extension $I(X, P)=1$ for all $P$ and (2.5) counts the number of representations of $\pi_{1} X$ into the finite group $G$. (There is an overall factor of $1 / \# G$.) In that case we can extend to a theory of unoriented manifolds. 
Now consider this 2-dimensional theory over the circle $S^{1}$. Recall from the introduction that the groupoid of $G$-bundles over $S^{1}$ is equivalent to $G / / G$, where $G$ acts on itself by conjugation. The value of the classical theory $I$ is given by the central extension $G^{\tau} \rightarrow G$, viewed as an equivariant principal $G$-bundle. In other words, the value $I\left(S^{1}, P\right)$ of this theory at a bundle with holonomy $x \in G$ is the circle torsor $G_{x}^{\tau}$ (together with the action of the centralizer of $x$, the automorphisms of $P$, by conjugation in the central extension.) In the quantum theory we make a sum of the corresponding hermitian lines $K_{P}$ analogous to (2.5) and so compute that $F\left(S^{1}\right)$ is the vector space of central sections of $K^{\tau} \rightarrow G$ :

$$
F\left(S^{1}\right)=\bigoplus_{P} \frac{1}{\# \operatorname{Aut} P}\left(K_{P}\right)^{\text {Aut } P}
$$

In this expression the metric on the hermitian line $K_{x}$ is scaled by the prefactor.

The quantum "path integrals" (2.5) and (2.6) may be expressed in categorical language. Let $\operatorname{Bord}_{2}^{S O}(G)$ denote the bordism category whose objects are closed oriented 1-manifolds equipped with a principal $G$-bundle and whose morphisms are compact oriented 2-manifolds equipped with a principal $G$-bundle. Then the classical topological theory $I$ is a 2-dimensional topological quantum field theory in the sense of Definition 1.1 with a special feature: its values are invertible. Thus the numbers attached to closed oriented surfaces with $G$-bundle are nonzero and the vector spaces attached to $G$-bundles over the circle are lines (which are invertible in the collection of vector spaces under tensor product). Now there is an obvious forgetful functor $\pi: \operatorname{Bord}_{2}^{S O}(G) \rightarrow \operatorname{Bord}_{2}^{S O}$ which omits the $G$-bundle. Then the quantum field theory $F$, obtained by summing over $G$-bundles, can be viewed as a kind of pushforward of $I$ along $\pi$. The relevant pushforward procedure has an analog in classical topology. Let $\pi: E \rightarrow S$ be a proper fiber bundle of topological manifolds whose fibers carry a suitable $n$-dimensional orientation. Then there is a map $\pi_{*}: h^{\bullet}(E) \rightarrow h^{\bullet-n}(S)$ variously termed the 'pushforward' or 'direct image' or 'wrong-way map' or 'umkehr map' or 'Gysin map' or 'transfer'. The analogy with our situation is tighter if we think of this pushforward on the level of cochains, or geometric representatives, rather than cohomology classes.

Formulas (2.5) and (2.6), together with a similar formula for compact surfaces with boundary, can be used to define the functor $F$. This constructs a 1-2 theory. We would like, however, to continue down to points, i.e., to a 0-1-2 theory. To express the higher gluing laws encoded in an $n$-dimensional TQFT which extends down to points we use the language of higher category theory; see $[\mathrm{L}$ for an introduction and for much more exposition about the $n$-categories we now introduce. Let $\operatorname{Bord}_{n}^{S O}$ denote the $n$-category 6 whose objects are finite unions of oriented points, 1-morphisms are oriented bordisms of objects, 2-morphisms are oriented bordisms of 1-morphisms, and so forth 7 Note $\operatorname{Bord}_{n}^{S O}$ carries a symmetric monoidal structure given by disjoint union. Let $\mathcal{C}$ be any symmetric monoidal $n$-category.

Definition 2.7. An extended $n$-dimensional TQFT with values in $\mathcal{C}$ is a symmetric monoidal functor $F: \operatorname{Bord}_{n}^{S O} \rightarrow \mathcal{C}$.

\footnotetext{
${ }^{6} \operatorname{Bord}_{n}^{S O}$ should be regarded as an $(\infty, n)$-category. This means roughly that we consider families of manifolds parametrized by a topological space rather than simply single manifolds.

${ }^{7} \mathrm{An}(\infty, n)$-category has $r$-morphisms for all $r$; they are invertible for $r>n$. The $r$-morphisms in Bord $\mathbf{B}_{n}^{S O}$ for $r>n$ are given by $(r-1)$-parameter families of diffeomorphisms of manifolds which preserve all boundaries (and corners).
} 
The 0-1-2 finite gauge theory can be constructed by hand.

\section{$\S 2.3$. 0-1-2 theories for general $G$}

Let $F$ be a fully extended topological field theory of dimension $n$. We can regard $F$ as a prescription for assigning invariants to manifolds with corners of all dimensions $\leqslant n$, together with set of rules for how these invariants behave when we glue manifolds together. Since every smooth manifold $M$ can be assembled by gluing together very simple pieces (for example, by choosing a triangulation of $M$ ), we might imagine that the value of $F$ on arbitrary manifolds is determined by its values on a very small class of manifolds. In order to formulate this idea more precisely, we need to introduce a bit of terminology.

Definition 2.8. Let $M$ be a manifold of dimension $m \leqslant n$. An $n$-framing of $M$ is a trivialization of the vector bundle $T_{M} \oplus \underline{\mathbb{R}^{n-m}}$, where $\underline{\mathbb{R}^{n-m}}$ denotes the trivial bundle (on $M$ ) of rank $n-m$. We let $\mathbf{B o r d}_{n}^{\mathrm{fr}}$ denote the bordism $n$-category whose $k$-morphisms are given by $n$-framed $k$-manifolds for $k \leqslant n$.

Theorem 2.9 (Baez-Dolan Cobordism Hypothesis). Let $\mathcal{C}$ be a symmetric monoidal n-category. Then the construction

$$
F \mapsto F(*)
$$

induces an injection from the collection of isomorphism classes of symmetric monoidal functors $F: \operatorname{Bord}_{n}^{\text {fr }} \rightarrow \mathcal{C}$ to the collection of isomorphism classes of objects of $\mathcal{C}$.

A version of Theorem 2.9 was originally conjectured by Baez and Dolan in [BD]. We refer the reader to $[\mathrm{L}]$ for a more extensive discussion and a sketch of the proof.

Let $\mathcal{C}$ be a symmetric monoidal $n$-category. We will say that an object $C \in \mathcal{C}$ is fully dualizable if there exists an extended TQFT $F: \mathbf{B o r d}_{n}^{\mathrm{fr}} \rightarrow \mathcal{C}$ and an isomorphism $C \simeq F(*)$. Theorem 2.9 asserts that if $C$ is a fully dualizable object of $\mathcal{C}$, then the field theory $F$ is uniquely determined by $C$.

Remark 2.10. It is possible to state a more precise version of Theorem 2.9 by describing the class of fully dualizable objects $C \in \mathcal{C}$ without mentioning the $n$-category $\operatorname{Bord}_{n}^{\text {fr }}$. If $n=1$, this condition is easy to state: the object $C$ should admit a dual $C^{\vee}$, so that there exist evaluation and coevaluation maps

$$
C \otimes C^{\vee} \stackrel{e}{\rightarrow} \mathbf{1} \quad \mathbf{1} \stackrel{c}{\rightarrow} C^{\vee} \otimes C
$$

(here 1 denotes the unit object of $\mathcal{C}$ ) which are compatible in the sense that the compositions

$$
\begin{gathered}
C \rightarrow C \otimes C^{\vee} \otimes C \rightarrow C \\
C^{\vee} \rightarrow C^{\vee} \otimes C \otimes C^{\vee} \rightarrow C^{\vee}
\end{gathered}
$$

both coincide with the identity. For $n>1$, we need to assume that analogous finiteness assumptions are satisfied not only by the object $C$, but by the 1-morphisms $e$ and $c$. We refer the reader to [L] for a more complete discussion. 
Theorem 2.9 has a curious consequence: since the orthogonal group $\mathrm{O}(n)$ acts on the $n$-category $\operatorname{Bord}_{n}^{\text {fr }}$ (by change of framing), we get an induced action of $\mathrm{O}(n)$ on the collection of fully dualizable objects of any symmetric monoidal $n$-category $\mathcal{C}$ (more precisely, the orthogonal group $\mathrm{O}(n)$ can be made to act on the classifying space for the underlying $n$-groupoid of the fully dualizable objects in $\mathcal{C})$. When $n=1$, this action is simply given by the involution that takes a dualizable object $C \in \mathcal{C}$ to its dual $C^{\vee}$.

We can use the action of the orthogonal group to formulate an analog of Theorem 2.9 for more general types of manifolds:

Theorem 2.11. Let $\mathcal{C}$ be a symmetric monoidal $n$-category. The construction $F \mapsto F(*)$ establishes a bijection between the set of isomorphism classes of symmetric monoidal functors Bord $_{n}^{\mathrm{SO}} \rightarrow \mathcal{C}$ with the set of isomorphism classes of (homotopy) fixed points for the action of the group $\operatorname{SO}(n)$ on the fully dualizable objects of $\mathcal{C}$.

Remark 2.12. Theorem 2.11 has an obvious analog for other types of manifolds: unoriented manifolds, spin manifolds, and so forth.

Example 2.13. Let $n=2$, and let $\mathcal{C}$ denote the 2-category $\mathcal{A l g}$ of (complex) algebras, bimodules, and intertwiners. Every object of $\mathcal{C}$ admits a dual: the dual of an algebra $A$ is the opposite algebra $A^{o p}$, where both the evaluation and coevaluation maps are given by $A$ (regarded as a $\left(\mathbb{C}, A \otimes A^{o p}\right)$ bimodule. An algebra $A$ is fully dualizable if and only if $A$ is dualizable both as a $\mathbb{C}$-module and as an $A \otimes A^{o p}$-module. The first condition amounts to the requirement that $A$ be finite dimensional over $\mathbb{C}$, while the second condition requires that the algebra $A$ be semisimple.

The circle group $\mathrm{SO}(2)$ acts on the classifying space of the 2-groupoid of fully dualizable objects of $\mathcal{A l g}$. In more concrete terms, this means that every fully dualizable object $A \in \mathcal{A l g}$ determines a functor from the fundamental 2-groupoid of $\mathrm{SO}(2)$ into $\mathcal{A l g}$ which carries the identity element of $\mathrm{SO}(2)$ to $A$. Applying this to a generator of the fundamental group $\pi_{1} \mathrm{SO}(2)$, we get an automorphism of $A$ in $\mathcal{A l g}$ : this automorphism is given by the vector space dual $A^{\vee}$, regarded as an $(A, A)$-bimodule. To realize $A$ as a fixed point for the action of $\mathrm{SO}(2)$, we need to choose an identification of $A$ with $A^{\vee}$ as $(A, A)$-bimodules. In other words, we need to choose a nondegenerate bilinear form $b: A \otimes A \rightarrow \mathbb{C}$ which satisfies the relations

$$
b\left(x a, a^{\prime}\right)=b\left(a, a^{\prime} x\right) \quad b\left(a x, a^{\prime}\right)=b\left(a, x a^{\prime}\right) .
$$

If we set $\operatorname{tr}(a)=b(1, a)$, then the first condition shows that $b\left(a, a^{\prime}\right)=\operatorname{tr}\left(a^{\prime} a\right)$, while the second condition shows that $b\left(a, a^{\prime}\right)=\operatorname{tr}\left(a a^{\prime}\right)$. It follows that $\operatorname{tr}$ is a trace on the algebra $A$ : that is, it vanishes on all commutators $\left[a, a^{\prime}\right]=a a^{\prime}-a^{\prime} a$. Conversely, given any linear map tr $: A \rightarrow \mathbb{C}$ which vanishes on all commutators, the formula $b\left(a, a^{\prime}\right)=\operatorname{tr}\left(a a^{\prime}\right)$ defines a bilinear form $b$ giving a map of $(A, A)$-bimodules $A \rightarrow A^{\vee}$. We say that tr is nondegenerate if this map is an isomorphism. A pair $(A, \operatorname{tr})$ where $A$ is a finite dimensional algebra over $\mathbb{C}$ and $\operatorname{tr}$ is a nondegenerate trace on $A$ is called a Frobenius algebra.

We can summarize the above discussion as follows: giving a fully dualizable object of $\mathcal{A l g}$ which is fixed under the action of the group $\mathrm{SO}(2)$ is equivalent to giving a semisimple Frobenius algebra $(A, \mathrm{tr})$. Theorem 2.11 implies that every such pair $(A, \operatorname{tr})$ determines an extended TQFT 
$F: \operatorname{Bord}_{2}^{\mathrm{SO}} \rightarrow \mathcal{A l g}$ such that $F(*) \simeq A$. The value of $F$ on the circle $S^{1}$ can be identified with the $\left(\mathbb{C}, \mathbb{C}\right.$ )-vector space given by the tensor product $A \otimes_{A \otimes A^{o p}} A$ : in other words, the quotient of $A$ by the subspace $[A, A]$ generated by all commutators. Using the self-duality of $F\left(S^{1}\right)$ provided by the field theory $F$ and the self-duality of $A$ provided by the bilinear form $b$, we can also identify $F\left(S^{1}\right)$ with the orthogonal $[A, A]^{\perp}$ of $[A, A]$ with respect to $b$ : that is, with the center of the algebra $A$.

In particular, if $G$ is a finite group and $\eta \in H^{3}(G ; \mathbb{Z})$ is a cohomology class, then the twisted group algebra $\mathbb{C}^{\tau}[G]$ admits a canonical trace (obtained by taking the coefficient of the unit in $G$ and dividing by the order of $G$ ). Applying Theorem 2.11 in this case, we obtain another construction of the topological field theory described in $\S 2.2$.

\section{Finite path integrals}

We sketch an idea to construct extended field theories via finite sums.

Remark 3.1. Integration over a finite field, such as a gauge field with finite gauge group, sometimes occurs in a quantum field theory with other fields. These cases also fit into this framework. Examples include orbifolds and orientifolds in string theory.

As in $[\mathrm{L}, \S 3.2]$ let $\mathrm{Fam}_{n}$ denote the $n$-category whose objects are finite groupoids $X$. (A groupoid $X$ is finite if there is a finite number of inequivalent objects and each object has a finite automorphism group.) A 1-morphism $C: X \rightarrow Y$ between finite groupoids is a correspondence

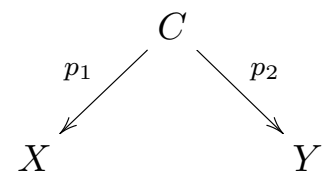

of finite groupoids. A 2-morphism in $\mathrm{Fam}_{n}$ is a correspondence of 1-morphisms, and so forth until the level $n$; we regard two $n$-morphisms in $\mathrm{Fam}_{n}$ as identical if they are equivalent. Composition is homotopy fiber product. Cartesian product of groupoids endows Fam $_{n}$ with a symmetric monoidal structure. There is a symmetric monoidal functor

$$
\operatorname{Bun}_{G}: \operatorname{Bord}_{n}^{S O} \longrightarrow \operatorname{Fam}_{n}
$$

which attaches to each manifold $M$ the finite groupoid of $G$-bundles on $M$. This is the space of classical fields on $M$. (The functor (3.3) replaces the category $\operatorname{Bord}_{n}^{S O}(G)$ which appears in our previous formulation in the paragraph following (2.6).) Let $\mathcal{C}$ be a symmetric monoidal $n$-category and $\operatorname{Fam}_{n}(\mathcal{C})$ the symmetric monoidal $n$-category of correspondences equipped with local systems valued in $\mathcal{C}$ : for example, an object of $\operatorname{Fam}_{n}(\mathcal{C})$ is a finite groupoid $X$ and a functor $X \rightarrow \mathcal{C}$, and morphisms are also equipped with functors to $\mathcal{C}$ (as before, $\mathcal{C}$ denotes the codomain of our 
field theories). Then the classical theory is encoded by a functor $I$ which fits into a commutative diagram

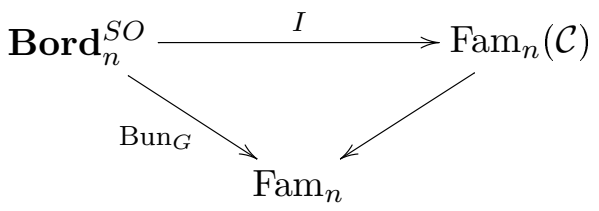

where the right arrow is the obvious forgetful functor.

Remark 3.4. For a classical theory the values of $I$ lie in the invertible objects and morphisms of $\mathcal{C}$. The finite sum construction does not depend on the invertibility, so will apply in the situations envisioned in Remark 3.1

Example 3.5. We recast the discussion in $\S 2.1$ in these terms. As a preliminary suppose $\mathcal{B}, \mathcal{C}$ are 2 -categories. We describe the data of a functor $I: \mathcal{B} \rightarrow \mathcal{C}$. Each 2-category consists of objects (ob), 1-morphisms (1-mor), and 2-morphisms (2-mor) and the data which defines $I$ includes a map between these corresponding collections in $\mathcal{B}$ and $\mathcal{C}$. For strict 2-categories these maps are required to respect the composition strictly. But for more general 2-categories there are two additional pieces of data. First, there is a map $u: \operatorname{ob}(\mathcal{B}) \rightarrow 2$-mor $(\mathcal{C})$ which for each object $X \in \operatorname{ob}(\mathcal{B})$ gives a 2-morphism $u(X): \operatorname{id}_{I(X)} \rightarrow I\left(\operatorname{id}_{X}\right)$. Second, there is a map $a: 1-\operatorname{mor}(\mathcal{B}) \times_{\mathrm{ob}(\mathcal{B})} 1$-mor $(\mathcal{B}) \rightarrow$ 2 -mor $(\mathcal{C})$ which expresses the failure of $I$ on 1-morphisms to be a strict homomorphism, namely $a(x, y): I(y) \circ I(x) \rightarrow I(y \circ x)$ for every pair $\stackrel{x}{\rightarrow} \cdot \stackrel{y}{\rightarrow} \cdot$ of composable 1 -morphisms in $\mathcal{B}$. These data are required to obey a variety of axioms. For example, if $\cdot \stackrel{x}{\rightarrow} \cdot \stackrel{y}{\rightarrow} \cdot \stackrel{z}{\rightarrow} \cdot$ is a triple of composable morphisms, then

$$
a(y \circ x, z) \circ\left\{a(x, y) * \operatorname{id}_{I(z)}\right\}=a(x, z \circ y) \circ\left\{\operatorname{id}_{I(x)} * a(y, z)\right\} .
$$

As in Example 2.13, let $\mathcal{C}=\mathcal{A l g}$ be the 2-category whose objects are complex algebras $A$. A morphism $A \rightarrow A^{\prime}$ is an $\left(A^{\prime}, A\right)$-bimodule and a 2-morphism is a homomorphism of bimodules. The symmetric monoidal structure is given by tensor product over $\mathbb{C}$. Note that the unit object in $\mathcal{C}$ is the algebra $\mathbb{C}$ and a $(\mathbb{C}, \mathbb{C})$-bimodule is simply a vector space. Thus the category of 1 -morphisms $\mathbb{C} \rightarrow \mathbb{C}$ is the category Vect $\mathbb{C}$ of complex vector spaces. Now for the finite 2-dimensional gauge theory based on the central extension $\mathbb{T} \rightarrow G^{\tau} \rightarrow G$, the functor $\operatorname{Bun}_{G}$ assigns to the point pt $t_{+}$the groupoid $* / / G$. The lift $I$ includes the functor

$$
\chi: * / / G \rightarrow \mathcal{A l g}
$$

which assigns the (invertible) algebra $\mathbb{C}$ to the unique object $*$, the complex line $K_{x}$ to the 1 morphism $x \in G$, and the identity map to the identity 2-morphisms in $* / / G$. (There are only identity 2-morphisms in $* / / G$.) The map $u$ in the previous paragraph is then the identity. The map $a$ in the previous paragraph assigns to each pair $x, y$ of group elements the isomorphism (2.2), viewed as a 2-morphism in $\mathcal{C}$. The associativity constraint (3.6) is (2.3). 
The quantization via a finite sum will be implemented by a symmetric monoidal functor of $n$-categories (see $8 \underline{8}$ )

$$
\operatorname{Sum}_{n}: \operatorname{Fam}_{n}(\mathcal{C}) \longrightarrow \mathcal{C}
$$

Given (3.8) we simply define the quantum theory as the composition

$$
F: \operatorname{Bord}_{n}^{S O} \stackrel{I}{\longrightarrow} \operatorname{Fam}_{n}(\mathcal{C}) \stackrel{\operatorname{Sum}_{n}}{\longrightarrow} \mathcal{C}
$$

The functor $\operatorname{Sum}_{n}$ depicted in (3.8) is given by a purely categorical procedure: if $X$ is a finite groupoid - an object in $\operatorname{Fam}_{n}$-and $\chi: X \rightarrow \mathcal{C}$ is a $\mathcal{C}$-valued local system on $X$, then $\operatorname{Sum}_{n}(X, \chi)$ is given by the colimit $\varliminf_{x \in X} \chi(x)$. To guarantee that this formula describes a well-defined functor from $\operatorname{Fam}_{n}(\mathcal{C})$ to $\mathcal{C}$, we need to make certain assumptions on $\mathcal{C}$ : namely, that it is additive in a strong sense which guarantees that the colimit $\lim _{x \in X} \chi(x)$ exists and coincides with the limit $\lim _{x \in X} \chi(x)$.

Remark 3.10. This discussion goes through if we replace $\mathrm{Fam}_{n}$ by the $n$-category whose objects are finite $n$-groupoids (see 98 ). We will use the generalization to $n=2$ in $\$ 5.2$.

Example 3.11. To illustrate the idea consider the 1-dimensional gauge theory of $\$ 1$ for a finite gauge group $G$. Recall it is specified by an abelian character $\lambda: G \rightarrow \mathbb{T}$. In this case let $\mathcal{C}$ be the symmetric monoidal category $V t_{\mathbb{C}}$ with tensor product. Then the "classical" functor $I: \operatorname{Bord}_{1}^{S O} \rightarrow \operatorname{Fam}_{1}\left(\right.$ Vect $\left._{\mathbb{C}}\right)$ sends the point $\mathrm{pt}_{+}$to the functor $* / / G \rightarrow \operatorname{Vect}_{\mathbb{C}}$ which sends $*$ to $\mathbb{C}$ and is the homomorphism $\lambda$ on morphisms. The path integral (3.8) is defined as follows. If $X \in \operatorname{Fam}_{1}\left(\right.$ Vect $\left._{\mathbb{C}}\right)$ is a finite groupoid equipped with a functor $\chi: X \rightarrow \operatorname{Vect}_{\mathbb{C}}$ then $\operatorname{Sum}_{1}(X, \chi) \in \operatorname{Vect}_{\mathbb{C}}$ is the limit

$$
\operatorname{Sum}_{1}(X, \chi)=\lim _{x \in X} \chi(x)
$$

If the finite groupoid $X=X_{0} \longleftarrow X_{1}$ is finitely presented ( $X_{0}$ and $X_{1}$ are finite sets), then $\chi$ determines an equivariant vector bundle over $X_{0}$ and the limit (3.12) is the vector space of invariant sections. On the other hand, a morphism in Fam $_{1}\left(\right.$ Vect $_{\mathbb{C}}$ ) is given by a correspondence (3.2) of finite groupoids; functors $\chi: X \rightarrow \operatorname{Vect}_{\mathbb{C}}$ and $\delta: Y \rightarrow \operatorname{Vect}_{\mathbb{C}}$; and for each $c \in C$ a linear map $\varphi(c): \chi\left(p_{1}(c)\right) \rightarrow \delta\left(p_{2}(c)\right)$. We define a map

$$
\operatorname{Sum}_{1}(C, \varphi): \operatorname{Sum}_{1}(X, \chi) \longrightarrow \operatorname{Sum}_{1}(Y, \delta)
$$

Assume $X, Y, C$ are finitely presented. Given $x \in X$ let $C_{x}$ be the sub-groupoid of $C$ consisting of $c \in C$ such that $p_{1}(c)=x$. Then

$$
\operatorname{Sum}_{1}(C, \varphi)=\sum_{[c] \in \pi_{0} C_{x}} \frac{\varphi(c)}{\# \operatorname{Aut}(c)}
$$

where the sum is over equivalence classes of objects in $C_{x}$. (Compare (2.5).) The reader can check that (3.12) and (3.13) reproduce the results in the paragraph preceding Remark 1.3 in the 1-dimensional finite gauge theory. 
Example 3.14. We continue Example 3.5 and content ourselves with the computation of $F\left(\mathrm{pt}_{+}\right)$ as a limit in the 2-category $\mathcal{C}=\mathcal{A l g}$ :

$$
F\left(p t_{+}\right)=\lim _{* / / G} \chi
$$

where $\chi$ is the functor (3.7). Unraveling the definitions, this limit is given by an algebra $A$ with the following universal property: for any algebra $B$, category of $(A, B)$-bimodules is equivalent to the category of right $B$-modules $M$ equipped with a compatible family of $B$-module isomorphisms $\left\{K_{x} \otimes M \simeq M\right\}_{x \in G}$. This limit can be represented by the twisted group algebra

$$
A=\mathbb{C}^{\tau}[G]=\bigoplus_{x \in G} K_{x}
$$

We leave as an exercise to the reader the computation of $F\left(S^{1}\right)$ as a (1-categorical) limit over the groupoid $G / / G$ of $G$-bundles on $S^{1}$. The argument is similar to Example 3.11.

\section{Three-dimensional theories with finite gauge group}

\section{$\S 4.1 . H^{4}(B G ; \mathbb{Z})$ for finite $G$}

Let $G$ be a finite group.

Definition 4.1. A 2-cocycle on $G$ with values in hermitian lines is a pair $\left(K^{\tau}, \theta^{\tau}\right)$ consisting of a hermitian line bundle $K^{\tau} \rightarrow G \times G$, for each triple $x, y, z \in G$ an isometry

$$
\theta_{x, y, z}: K_{y, z} \otimes K_{x y, z}^{-1} \otimes K_{x, y z} \otimes K_{x, y}^{-1} \longrightarrow \mathbb{C}
$$

and a cocycle condition

$$
\theta_{y, z, w} \theta_{x y, z, w}^{-1} \theta_{x, y z, w} \theta_{x, y, z w}^{-1} \theta_{x, y, z}=1, \quad x, y, z, w \in G
$$

for each quadruple of elements of $G$.

Proposition 4.4. For $G$ finite the cohomology group $H^{4}(B G ; \mathbb{Z})$ is the set of isomorphism classes of 2-cocycles $\left(K^{\tau}, \theta^{\tau}\right)$ on $G$ with values in hermitian lines.

Proof. Since $H^{4}(B G ; \mathbb{R})=0$, the Bockstein homomorphism $H^{3}(B G ; \mathbb{T}) \rightarrow H^{4}(B G ; \mathbb{Z})$ from the exponential sequence of coefficients is an isomorphism 8 Given $\left(K^{\tau}, \theta^{\tau}\right)$ choose $k_{x, y} \in K_{x, y}$ of unit norm. Then

$$
\omega_{x, y, z}=\theta_{x, y, z}\left(k_{y, z} k_{x y, z}^{-1} k_{x, y z} k_{x, y}^{-1}\right)
$$

\footnotetext{
${ }^{8}$ Here the circle $\mathbb{T}$ has either the discrete topology or the continuous topology.
} 
is a 3 -cocycle with values in $\mathbb{T}$. A routine check shows that the resulting element of $H^{3}(B G ; \mathbb{T})$ is independent of $\left\{k_{x, y}\right\}$ and of the representative $\left(K^{\tau}, \theta^{\tau}\right)$ in an equivalence class. Also, the resulting map from equivalence classes of 2-cocycles to $H^{3}(B G ; \mathbb{T})$ is an injective homomorphism. Surjectivity is also immediate: if $\omega_{x, y, z}$ is a cocycle, set $K_{x, y}=\mathbb{C}$ and $\theta_{x, y, z}=\omega_{x, y, z}$.

Suppose $\left(K^{\tau}, \theta^{\tau}\right)$ is given. Define the line bundle $L \rightarrow G \times G$ by

$$
L_{x, y}=K_{y x y^{-1}, y}^{*} \otimes K_{y, x} .
$$

The cocycle isomorphism (4.2) leads to an isomorphism

$$
L_{y x y^{-1}, y^{\prime}} \otimes L_{x, y} \longrightarrow L_{x, y^{\prime} y}
$$

which is summarized by the statement that $L$ is a line bundle over the groupoid $G / / G$ formed by the $G$ action on itself by conjugation. In $\S 4.2$ the line bundle $K$ enters into the quantization of a point and the line bundle $L$ enters into the quantization of the circle.

\section{$\S 4.2$. Three-dimensional finite gauge theory}

Let $G$ be finite and fix a 2-cocycle $\left(K^{\tau}, \theta^{\tau}\right)$ with values in hermitian lines. Let $\operatorname{Vect}^{\tau}[G]$ be the category whose objects are complex vector bundles over $G$ and morphisms are linear vector bundle maps. Define a monoidal structure on $\operatorname{Vect}^{\tau}[G]$ by twisted convolution: if $W, W^{\prime} \rightarrow G$ are vector bundles set

$$
\left(W * W^{\prime}\right)_{y}=\bigoplus_{x x^{\prime}=y} K_{x, x^{\prime}} \otimes W_{x} \otimes W_{x^{\prime}}^{\prime}
$$

Then $\operatorname{Vect}^{\tau}[G]$ is a linear tensor category.

Remark 4.6. One way to regard this category is as the "crossed product" $T \ltimes$ Vect, with the tensor category Vect replacing the customary algebra with $T$-action. (Actions of $T$ on Vect, as a tensor category, assign to each $t \in T$ an invertible (Vect, Vect)-bimodule, which must of course be isomorphic to Vect. The associator for the action is a 2-cocycle on $T$ with values in Pic, the group of units in Vect; and associators for equivalent actions differ by a co-boundary. Our action is classified by $\tau$, as per the discussion in $\$ 4.1$.)

There is a TQFT $F^{\tau}$ such that $F^{\tau}(\mathrm{pt})=\operatorname{Vect}{ }^{\tau}[G]$ and it can be constructed in several ways. First, and most directly, we can construct it as a 0-1-2-3 theory directly using the finite sum path integral in 93 . Secondly, following Reshetikhin and Turaev [T], as a 1-2-3 theory it may be constructed by specifying the modular tensor category which is attached to a circle; it appears in Proposition 4.9 below. A third approach is to realize $\operatorname{Vect}^{\tau}[G]$ as a fully dualizable object of a symmetric monoidal 3-category $\mathcal{C}$. The Baez-Dolan cobordism hypothesis then implies that $\operatorname{Vect}^{\tau}[G]$ determines a 0-1-2-3 theory which is defined on framed 3-manifolds. To remove the dependence on a choice of framing, we should go further and exhibit $\operatorname{Vect}^{\tau}[G]$ as an $S O(3)$-equivariant fully dualizable object. For present purposes, we will be content to sketch a definition of the relevant 3 -category $\mathcal{C}$ and to give some hints at what the relevant finiteness conditions correspond to. 
Definition 4.7. The 3 -category $\mathcal{C}$ can be described informally as follows:

(a) The objects of $\mathcal{C}$ are tensor categories over $\mathbb{C}$ : in other words, $\mathbb{C}$-linear categories equipped with a tensor product operation which is associative up to coherent isomorphism.

(b) Given a pair of tensor categories $\mathscr{A}$ and $\mathscr{A}^{\prime}$, a 1-morphism from $\mathscr{A}$ to $\mathscr{A}^{\prime}$ in $\mathcal{C}$ is an $\mathscr{A}$ - $\mathscr{A}^{\prime}$ bimodule category: that is, a $\mathbb{C}$-linear category $\mathcal{D}$ equipped with a left action $\mathscr{A} \times \mathcal{D} \rightarrow \mathcal{D}$ and a right action $\mathcal{D} \times \mathscr{A}^{\prime} \rightarrow \mathcal{D}$ which commute with one another, up to coherent isomorphism.

(c) Given a pair of tensor categories $\mathscr{A}$ and $\mathscr{A}^{\prime}$ and a pair of bimodule categories $\mathcal{D}$ and $\mathcal{D}^{\prime}$, a 2-morphism from $\mathcal{D}$ to $\mathcal{D}^{\prime}$ in $\mathcal{C}$ is a functor between bimodule categories: that is, a functor $F: \mathcal{D} \rightarrow \mathcal{D}^{\prime}$ which commutes with the actions of $\mathscr{A}$ and $\mathscr{A}^{\prime}$ up to coherent isomorphism.

(d) Given a pair of tensor categories $\mathscr{A}$ and $\mathscr{A}^{\prime}$, a pair of bimodule categories $\mathcal{D}$ and $\mathcal{D}^{\prime}$, and a pair of bimodule category functors $F, F^{\prime}: \mathcal{D} \rightarrow \mathcal{D}^{\prime}$, a 3-morphism from $F$ to $F^{\prime}$ in $\mathcal{C}$ is a natural transformation $\alpha: F \rightarrow F^{\prime}$ which is compatible with the coherence isomorphisms of (c).

The category $\operatorname{Vect}^{\tau}[G]$ is a fully dualizable object of $\mathcal{C}$. Roughly speaking, the verification of this takes place in three (successively more difficult) steps. First, we verify that $\operatorname{Vect}^{\tau}[G]$ is dualizable: in other words, that it is a fully dualizable object of the underlying 1-category of $\mathcal{C}$ (and therefore gives rise to a 1-dimensional field theory). This is completely formal: every tensor category $\mathscr{A}$ is a dualizable object of $\mathcal{C}$, the dual being the same category with the opposite tensor product. The next step is to verify that $\operatorname{Vect}^{\tau}[G]$ is a fully dualizable object of the underlying 2-category of $\mathcal{C}$ (and therefore gives rise to a 2-dimensional field theory, which assigns vector spaces to surfaces). This is a consequence of the fact that $\operatorname{Vect}^{\tau}[G]$ is a rigid tensor category: that is, every object of $X \in \operatorname{Vect}^{\tau}[G]$ has a dual (given by taking the dual vector bundle $X^{\vee}$ of $X$ and pulling back under the inversion map $g \mapsto g^{-1}$ from $G$ to itself). Finally, to get a 3-dimensional field theory, we need to check that $\operatorname{Vect}^{\tau}[G]$ satisfies some additional 3-categorical finiteness conditions which we will not spell out here. (However, we should remark that these 3-categorical finiteness conditions are in some sense the most concrete, and often amount to the finite dimensionality of various vector spaces associated to $\operatorname{Vect}^{\tau}[G]$ : for example, the vector spaces of morphisms between objects of $\operatorname{Vect}^{\tau}[G]$.) The paper [BW] is presumably relevant to the full dualizability of $\operatorname{Vect}^{\tau}[G]$.

Definition 4.8. Let $\mathscr{A}$ be a monoidal category with product $*$. Its (Drinfeld) center $Z(\mathscr{A})$ is the category whose objects are pairs $\left(X, \epsilon_{X}\right)$ consisting of an object $X$ in $\mathscr{A}$ and a natural isomorphism $\epsilon_{X}(-): X *-\rightarrow-* X$. The isomorphism $\epsilon_{X}$ is compatible with the monoidal structure in that for all objects $Y, Z$ in $\mathscr{A}$ we require

$$
\epsilon_{X}(Y * Z)=\left(\operatorname{id}_{Y} * \epsilon_{X}(Z)\right) \circ\left(\epsilon_{X}(Y) * \operatorname{id}_{Z}\right)
$$

The center $Z(\mathscr{A})$ of any monoidal category $\mathscr{A}$ is a braided monoidal category. Müger [M] proves that if $\mathscr{A}$ is a linear tensor category over an algebraically closed field which satisfies certain conditions, then $Z(\mathscr{A})$ is a modular tensor category. That applies to part (ii) of the following result.

Proposition 4.9. (i) The value $F^{\tau}\left(S^{1}\right)$ of the field theory $F^{\tau}$ on the circle is the center of the monoidal category $\operatorname{Vect}^{\tau}[G]$. 
(ii) The center of $\operatorname{Vect}^{\tau}[G]$ consists of twisted equivariant vector bundles $W \rightarrow G$, that is, vector bundles with a twisted lift

$$
L_{x, y} \otimes W_{x} \longrightarrow W_{y x y^{-1}}
$$

of the $G$-action on $G$ by conjugation, where $L \rightarrow G \times G$ is defined in (4.5).

This center is the well-known modular tensor category attached to a circle in the twisted finite group Chern-Simons theory [F2].

Proof. We compute $F^{\tau}\left(S^{1}\right)$ by decomposing the circle into two intervals $I_{L}$ and $I_{R}$. We regard $I_{L}$ as a morphism in the oriented bordism category from the empty set to the disjoint union of two oppositely oriented points; $I_{R}$ is a bordism in the other direction. Then $S^{1}$ is the composition $I_{R} \circ I_{L}$. Let $A=\operatorname{Vect}^{\tau}[G]$, which is the object in $\mathcal{C}$ - a tensor category - attached to a positively oriented point. Then the tensor category $A^{\mathrm{op}}$ with the opposite monoidal structure is attached to the negatively oriented point. Now $F^{\tau}\left(I_{L}\right)$ is $A$ viewed as a left module for $A \otimes A^{\text {op }}$ and $F^{\tau}\left(I_{R}\right)$ is $A$ viewed as a right module for $A \otimes A^{\text {op }}$. Thus

$$
F^{\tau}\left(S^{1}\right) \cong F^{\tau}\left(I_{R} \circ I_{L}\right) \cong A \otimes_{A \otimes A^{\text {op }}} A
$$

(This is by definition the Hochschild homology of $A$.) But now we use additional structure on $\operatorname{Vect}^{\tau}[G]$ which gives an isomorphism of $A$ with its linear dual $A^{\vee}=\operatorname{Hom}(A$, Vect). Namely, there is a trace $\theta: A \rightarrow$ Vect which maps a vector bundle over $G$ to the fiber over the identity element, and the corresponding bilinear form

$$
\begin{aligned}
A \otimes A & \longrightarrow \text { Vect } \\
W \otimes W^{\prime} & \longmapsto \theta\left(W * W^{\prime}\right)
\end{aligned}
$$

induces the desired identification. Therefore,

$$
F^{\tau}\left(S^{1}\right) \cong A \otimes_{A \otimes A^{\mathrm{op}}} A^{\vee} \cong \operatorname{Hom}_{A \otimes A^{\mathrm{op}}}(A, A)
$$

which we may identify with the center of $A$ (the Hochschild cohomology).

For (ii) suppose $W \rightarrow G$ is in the center. Let $W^{\prime} \rightarrow G$ be the vector bundle which is the trivial line $\mathbb{C}_{y}$ at some $y \in G$ and zero elsewhere. Then the braiding gives, for every $x \in G$, an isomorphism

$$
K_{y, x} \otimes \mathbb{C}_{y} \otimes W_{x} \longrightarrow K_{y x y^{-1}, y} \otimes W_{y x y^{-1}} \otimes \mathbb{C}_{y}
$$

which, by (4.5), is the desired isomorphism (4.10). 


\section{2-cocycles on tori}

\section{$\S 5.1 . H^{4}(B G ; \mathbb{Z})$ for torus groups}

Let $G=T$ be a compact connected abelian Lie group, i.e., a torus. Associated to it are the dual lattices (finitely generated free abelian groups)

$$
\begin{aligned}
& \Pi=\operatorname{Hom}(\mathbb{T}, T) \cong H_{1}(T ; \mathbb{Z}) \cong H_{2}(B T ; \mathbb{Z}) \\
& \Lambda=\operatorname{Hom}(T, \mathbb{T}) \cong H^{1}(T ; \mathbb{Z}) \cong H^{2}(B T ; \mathbb{Z})
\end{aligned}
$$

Let $\mathfrak{t}$ be the Lie algebra of $T$. Then $\Pi \subset \mathfrak{t}$ by differentiation of a homomorphism, and dually $\Lambda \subset \mathfrak{t}^{*}$, also by differentiation. The cohomology ring of $B T$ is the symmetric ring on $H^{2}(B T ; \mathbb{Z})=\Lambda$, so in particular

$$
H^{4}(B T ; \mathbb{Z}) \cong \operatorname{Sym}^{2} \Lambda
$$

We identify $\operatorname{Sym}^{2} \Lambda$ with the group of homogeneous quadratic functions $q: \Pi \rightarrow \mathbb{Z}$. For there is a natural quotient map $\Lambda^{\otimes 2} \rightarrow \operatorname{Sym}^{2} \Lambda$ with kernel the alternating tensors, and the value $q(\pi)$ of the lift of an element of $\operatorname{Sym}^{2} \Lambda$ on $\pi \otimes \pi$ is independent of the lift. Then the symmetric bi-additive homomorphism (form)

$$
\left\langle\pi_{1}, \pi_{2}\right\rangle=q\left(\pi_{1}+\pi_{2}\right)-q\left(\pi_{1}\right)-q\left(\pi_{2}\right), \quad \pi_{1}, \pi_{2} \in \Pi,
$$

is even $(\langle\pi, \pi\rangle \in 2 \mathbb{Z})$ and $q(\pi)=\frac{1}{2}\langle\pi, \pi\rangle$. Therefore, the group $\operatorname{Sym}^{2} \Lambda$ in (5.1) is also isomorphic to the group of even forms $\Pi \times \Pi \rightarrow \mathbb{Z}$.

Definition 5.3. A class in $H^{4}(B T ; \mathbb{Z})$ is nondegenerate if the corresponding form $\langle-,-\rangle$ is nondegenerate over $\mathbb{Q}$.

The form induces a homomorphism $\tau: \Pi \rightarrow \Lambda$; it is nondegenerate over $\mathbb{Q}$ if the map $\tau_{\mathbb{Q}}: \Pi \otimes \mathbb{Q} \rightarrow$ $\Lambda \otimes \mathbb{Q}$ on rational vector spaces is an isomorphism, or equivalently if $\tau$ is injective.

Remark 5.4. The group $\left(\mathrm{Sym}^{2} \Pi\right)^{*}$ of all symmetric bi-additive homomorphisms $\Pi \times \Pi \rightarrow \mathbb{Z}$ fits into the exact sequence

$$
0 \longrightarrow \operatorname{Sym}^{2} \Lambda \longrightarrow\left(\operatorname{Sym}^{2} \Pi\right)^{*} \longrightarrow \operatorname{Hom}(\Pi, \mathbb{Z} / 2 \mathbb{Z}) \longrightarrow 0
$$

where the quotient map takes a form $\langle-,-\rangle$ to $\pi \mapsto\langle\pi, \pi\rangle(\bmod 2)$. The quotient is the cohomology group $H^{2}(B T ; \mathbb{Z} / 2 \mathbb{Z})$, the kernel is the cohomology group $H^{4}(B T ; \mathbb{Z})$, and we can identify the middle term with the cohomology group $h^{4}(B T)$, where $h$ is the first cohomology theory mentioned in Remark 1.3.

Fix a nondegenerate class in $H^{4}(B T ; \mathbb{Z})$ with corresponding form $\langle-,-\rangle$ and homomorphism $\tau: \Pi \rightarrow \Lambda$. Applying $\otimes \mathbb{R}$ we extend the form to $\mathfrak{t} \times \mathfrak{t}$ and obtain a linear map $\tau_{\mathbb{R}}: \mathfrak{t} \rightarrow \mathfrak{t}^{*}$. The 
nondegeneracy implies that $\tau_{\mathbb{R}}$ is invertible. Let $L \rightarrow \mathfrak{t} \times \mathfrak{t}$ be the trivial line bundle and lift the action of $\Pi \times \Pi$ on $\mathfrak{t} \times \mathfrak{t}$ to $L$ by setting

$$
\left(\pi, \pi^{\prime}\right): L_{\xi, \xi^{\prime}} \longrightarrow L_{\xi+\pi, \xi^{\prime}+\pi^{\prime}}, \quad \pi, \pi^{\prime} \in \Pi, \quad \xi, \xi^{\prime} \in \mathfrak{t}
$$

to act as multiplication by

$$
e\left(\frac{\left\langle\pi, \xi^{\prime}\right\rangle-\left\langle\xi, \pi^{\prime}\right\rangle+\left\langle\pi, \pi^{\prime}\right\rangle}{2}\right)
$$

where $e(a)=e^{2 \pi i a}$ for a real number $a$. Also, define the correspondence

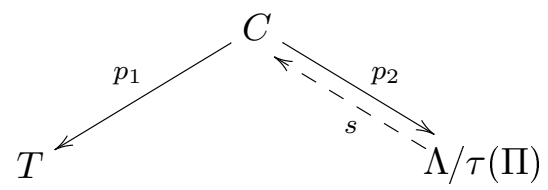

by $C=(\mathfrak{t} \oplus \Lambda) / \Pi$ where the action by $\pi \in \Pi$ on $(\xi, \lambda) \in \mathfrak{t} \times \Lambda$ is

$$
\pi \cdot(\xi, \lambda)=(\xi+\pi, \lambda+\tau(\pi))
$$

Proposition 5.9. (i) The expression (5.6) does lift the action of $\Pi \times \Pi$, whence there is a quotient hermitian line bundle $L \rightarrow T \times T$. There are natural isomorphisms

$$
L_{x, y^{\prime}} \otimes L_{x, y} \longrightarrow L_{x, y^{\prime} y}, \quad x, y, y^{\prime} \in T
$$

which lift to the identity map on $\mathfrak{t}^{\times 3}$ and which satisfy an associativity condition. Hence for each $x \in T$ the bundle $L_{x,-} \rightarrow T$ determines a central extension $\mathbb{T} \rightarrow \hat{T}_{x} \rightarrow T$.

(ii) The fiber of $p_{1}$ over $x \in T$ may be identified with the $\Lambda$-torsor of splittings of $\mathbb{T} \rightarrow \hat{T}_{x} \rightarrow T$. The splitting $\chi_{(\xi, \lambda)}$ corresponding to $(\xi, \lambda) \in \mathfrak{t} \times \Lambda$ is determined by

$$
\chi_{(\xi, \lambda)}\left(\xi^{\prime}\right)=e\left(\left\langle\tau_{\mathbb{R}}^{-1}(\lambda)-\frac{\xi}{2}, \xi^{\prime}\right\rangle\right) \in L_{\xi, \xi^{\prime}}, \quad \xi^{\prime} \in \mathfrak{t}
$$

(iii) $C$ is a group and $p_{2}$ is split by the homomorphism $s: \Lambda / \tau(\Pi) \rightarrow C$ defined by

$$
s(\lambda)=\left(\tau_{\mathbb{R}}^{-1}(\lambda), \lambda\right) \in \mathfrak{t} \oplus \Lambda, \quad \lambda \in \Lambda .
$$

The proof is a series of straightforward verifications from (5.6), (5.8), (5.11), and (5.12).

Notation 5.13. Let $\hat{F} \subset C$ denote the image of $s$ and $F \subset T$ the image $p_{1}(\hat{F})$. 
Both $F$ and $\hat{F}$ are finite groups isomorphic to $\Lambda / \tau(\Pi)$. The abelian groups $F$ and $\Lambda / \tau(\Pi)$ are in Pontrjagin duality by pairing characters in $\Lambda$ with elements of $F \subset T$. The map $p_{2}$ has as fibers affine spaces for $\mathfrak{t}$. The subgroup $\hat{F}$ contains a unique element in each affine space. Also, for each $x \in F$ the lift $\hat{x} \in \hat{F} \subset C$ defines a distinguished projective character of $\hat{T}_{x}$. Observe that $\mathfrak{t} \subset C$ as the fiber of $p_{2}$ over 0 , and $C$ is isomorphic to the direct sum $\mathfrak{t} \oplus \hat{F}$.

A class in $H^{4}(B T)$ may also be represented by a 2-cocycle 9 , as in Proposition 4.4, and it has an explicit construction analogous to that of $L$ in (5.5). It depends on a choice of a (nonsymmetric) bilinear form $B: \Pi \times \Pi \rightarrow \mathbb{Z}$ which whose symmetrization is the form (5.2):

$$
B\left(\pi_{1}, \pi_{2}\right)+B\left(\pi_{2}, \pi_{1}\right)=\left\langle\pi_{1}, \pi_{2}\right\rangle, \quad \pi_{1}, \pi_{2} \in \Pi
$$

Namely, let $K \rightarrow \mathfrak{t} \times \mathfrak{t}$ be the trivial line bundle and lift the action of $\Pi \times \Pi$ on $\mathfrak{t} \times \mathfrak{t}$ to $K$ by setting

$$
\left(\pi, \pi^{\prime}\right): K_{\xi, \xi^{\prime}} \longrightarrow K_{\xi+\pi, \xi^{\prime}+\pi^{\prime}}, \quad \pi, \pi^{\prime} \in \Pi, \quad \xi, \xi^{\prime} \in \mathfrak{t}
$$

to act as multiplication by

$$
e\left(\frac{B\left(\pi, \xi^{\prime}\right)-B\left(\xi, \pi^{\prime}\right)+B\left(\pi, \pi^{\prime}\right)}{2}\right)
$$

Proposition 5.16. (i) The expression (5.15) does lift the action of $\Pi \times \Pi$, whence there is a quotient hermitian line bundle $K \rightarrow T \times T$. There are natural isomorphisms $\theta_{x, y, z}$ as in (4.2) which lift to the identity map on $\mathfrak{t}^{\times 3}$ and which satisfy the cocycle condition (4.3).

(ii) There is an isomorphism

$$
L_{x, y} \stackrel{\cong}{\rightrightarrows} K_{x, y}^{-1} \otimes K_{y, x}
$$

such that (5.10) is $\theta_{x, y^{\prime}, y} \theta_{y^{\prime}, y, x} \theta_{y^{\prime}, x, y}^{-1}$.

The proof is a series of straightforward verifications.

\section{$\S 5.2$. Classical descriptions}

We continue with the notation of $\$ 5.1$. Recall that the level is a class in $H^{4}(B T ; \mathbb{Z})$ and is represented by a homogeneous quadratic map $q: \Pi \rightarrow \mathbb{Z}$. It determines a homomorphism $\tau: \Pi \rightarrow \Lambda$. The finite subgroup $F \subset T$, defined in Notation [5.13, may be identified as

$$
F \cong \tau_{\mathbb{Q}}^{-1}(\Lambda) / \Pi \subset(\Pi \otimes \mathbb{Q}) / \Pi
$$

Then $q$ induces a homogeneous quadratic map $q_{\mathbb{Q} / \mathbb{Z}}: F \rightarrow \mathbb{Q} / \mathbb{Z}$. We give a topological interpretation. For an abelian group $A$ and nonnegative integer $n$, let $K(A, n)$ be the corresponding EilenbergMacLane space.

\footnotetext{
${ }^{9}$ A 2-cocycle on $T$ with values in hermitian lines is defined as in Definition 4.1 with the additional requirement that the line bundle $K \rightarrow T \times T$ and isometry (4.2) be smooth.
} 
Lemma 5.17 ([EL, Theorem 26.1]). Let $A, B$ be discrete abelian groups. Then the set of homogeneous quadratic forms $q: A \rightarrow B$ is isomorphic to the homotopy classes of maps $\tilde{q}: K(A, 2) \rightarrow$ $K(B, 4)$.

Applying the lemma we may represent $q_{\mathbb{Q} / \mathbb{Z}}$ by a continuous map

$$
\tilde{q}_{\mathbb{Q} / \mathbb{Z}}: K(F, 2) \longrightarrow K(\mathbb{Q} / \mathbb{Z}, 4)
$$

Now for any manifold $X$ let $\mathcal{F}_{F}(X)$ denote the 2 -groupoid of " $F$-gerbes" on $X$. Recall that an $F$-gerb 10 is a geometric representative of a class in $H^{2}(X ; F)$. One possible topological model is that an $F$-gerbe on $X$ is a map $X \rightarrow K(F, 2)$. In this model $\mathcal{F}_{F}(X)$ is the fundamental 2-groupoid of the mapping space $\operatorname{Map}(X, K(F, 2))$. There are three nonzero homotopy groups:

$$
\pi_{0} \mathcal{F}_{F}(X) \cong H^{2}(X ; F), \quad \pi_{1} \mathcal{F}_{F}(X) \cong H^{1}(X ; F), \quad \pi_{2} \mathcal{F}_{F}(X) \cong H^{0}(X ; F)
$$

Composition with (5.18) gives the lagrangian of the field theory. Suppose $X$ is a closed oriented 4manifold. Then the action is defined by integrating the lagrangian, which in this case means pairing with the fundamental class given by the orientation. The result only depends on the equivalence class of the gerbe and gives a homogeneous quadratic map

$$
q_{X}: H^{2}(X ; F) \longrightarrow \mathbb{Q} / \mathbb{Z}
$$

which is defined using the cup square and the quadratic form $q$. The quantum invariant is then a finite path integral ( 93 ) over the stack of $F$-gerbes:

$$
\mathscr{A}_{F}(X)=\sum_{\mathcal{G}} \frac{\# H^{0}(X ; F)}{\# H^{1}(X ; F)} e^{2 \pi i q_{X}(\mathcal{G})},
$$

where the sum is over a set of representative F-gerbes on $X$. (Compare (2.5).) This Gauss sum may be evaluated explicitly $[\mathrm{HM}]$ :

$$
\begin{aligned}
\mathscr{A}_{F}(X) & =\frac{\# H^{0}(X ; F)}{\# H^{1}(X ; F)} \sqrt{\# H^{2}(X ; F)} \exp [2 \pi i(\operatorname{sign} b)(\operatorname{sign} X) / 8] \\
& =(\sqrt{\# F})^{\text {Euler } X} \mu^{(\operatorname{sign} b)(\operatorname{sign} X)},
\end{aligned}
$$

where $\operatorname{sign} X$ is the signature and Euler $X$ the Euler characteristic of the 4-manifold $X$; $\operatorname{sign} b$ is the signature of the bilinear form $b=\langle-,-\rangle$ in (5.2) associated to $q$ (after tensoring with $\mathbb{Q}$ ); and $\mu=\exp (2 \pi i / 8)$ is a primitive $8^{\text {th }}$ root of unity.

We claim that the finite path integral procedure of 93 , applied to the $F$-gerbes and the quadratic form $q$, defines an invertible 4-dimensional TQFT $\mathscr{A}_{F}$. For this we need to first specify a target

\footnotetext{
${ }^{10}$ Physicists are familiar with gerbes as fields in a field theory in string theories, where they are known as " $B$-fields".
} 
symmetric monoidal 4-category $\mathcal{C}$. The relevant category can be described informally as a "delooping" of the 3-category of Definition 4.7. Roughly speaking, the objects of $\mathcal{C}$ are braided tensor categories $\mathscr{A}$ (which we can think of as associative algebras in the setting of tensor categories). A 1-morphism from $\mathscr{A}$ to $\mathscr{A}^{\prime}$ is an $\mathscr{A}-\mathscr{A}^{\prime}$ bimodule in the setting of tensor categories: that is, a tensor category $\mathcal{D}$ equipped with commuting central actions of the braided monoidal categories $\mathscr{A}$ and $\mathscr{A}^{\prime}$. The 2-morphisms in $\mathcal{C}$ are given by linear categories, the 3 -morphisms by functors, and the 4-morphisms by natural transformations. (See $\$ 7.2$ for further discussion.)

Here we give a second classical description of the anomaly theory which leads to a finite dimensional but not finite path integral, and we use some heuristics in its evaluation on a 4-manifold. In this theory the finite group $F$ is replaced by the Lie algebra $\mathfrak{t}$ with the discrete topology. Now we use the real homogeneous quadratic form $q_{\mathbb{R}}: \mathfrak{t} \rightarrow \mathbb{R}$ and apply Lemma 5.17 to obtain

$$
K(\mathfrak{t}, 2) \longrightarrow K(\mathbb{R}, 4) \longrightarrow K(\mathbb{R} / \mathbb{Z}, 4)
$$

Let $\mathcal{F}_{\mathfrak{t}}(X)$ be the 2-groupoid of $\mathfrak{t}$-gerbes on a closed oriented 4-manifold $X$. Then (5.21) determines a homogeneous quadratic map

$$
q_{X}: H^{2}(X ; \mathfrak{t}) \longrightarrow \mathbb{R} / \mathbb{Z}
$$

The finite sum (5.19) is now replaced by a sum over an uncountable set, which we interpret as an integral:

$$
\mathscr{A}_{\mathfrak{t}}(X)=\int_{\mathcal{G} \in H^{2}(X ; \mathfrak{t})} \frac{\operatorname{vol} H^{0}(X ; \mathfrak{t})}{\operatorname{vol} H^{1}(X ; \mathfrak{t})} e^{2 \pi i q_{X}(\mathcal{G})} .
$$

Here 'vol' denotes a formal volume which we regularize below. This is a Gaussian integral, and we evaluate it a: 11

$$
\begin{aligned}
\mathscr{A}_{\mathfrak{t}}(X) & =\frac{\operatorname{vol} H^{0}(X ; \mathfrak{t})}{\operatorname{vol} H^{1}(X ; \mathfrak{t})} \sqrt{\operatorname{vol} H^{2}(X ; \mathfrak{t})} \exp [2 \pi i(\operatorname{sign} b)(\operatorname{sign} X) / 8] \\
& =\lambda^{\text {Euler } X} \mu^{(\operatorname{sign} b)(\operatorname{sign} X)},
\end{aligned}
$$

where $\lambda$ is a constant we choose equal to $\sqrt{\# F}$ to match (5.20).

Before leaving these classical descriptions we indicate a classical coupling of the usual toral Chern-Simons to the classical gerbe theory $\mathscr{A}_{\mathfrak{t}}$ on a compact oriented 4-manifold $X$ with boundary a closed oriented 3-manifold $Y$. We freely use generalized differential cohomology [HS] 12 Let

\footnotetext{
${ }^{11}$ If $Q$ is a symmetric bilinear form on a finite dimensional real vector space $V$, it induces a map $V \rightarrow V^{*}$ whose determinant is a map $\operatorname{Det} Q: \operatorname{Det} V \rightarrow \operatorname{Det} V^{*}$, so an element $\operatorname{Det} Q \in\left(\operatorname{Det} V^{*}\right)^{\otimes 2}$. The integral of $e^{i Q(x, x) / 2}$ over $V$ has an algebraic evaluation as$$
\frac{e^{2 \pi i(\operatorname{sign} Q) / 8}}{\sqrt{|\operatorname{det} Q|}} \in|\operatorname{Det} V|,
$$

where $\mid$ Det $V \mid$ is the real line associated to $V$ by the absolute value character of $\mathbb{R}^{\neq 0}$. A translation-invariant volume form on $V$ may be viewed as an element of the dual real line $\mid$ Det $V^{*} \mid$, which then gives a numerical answer which matches the usual Gaussian integral. This explains the signature factors in (5.23).

${ }^{12}$ We use differential theories based on the Eilenberg-MacLane spectrum $H \Pi$.
} 
$\mathcal{G}$ be a $\mathfrak{t}$-gerbe on $X$; since $\mathfrak{t}$ has the discrete topology $\mathcal{G}$ is flat. Its restriction $\partial \mathcal{G}$ to $Y$ has an exponential $\exp \partial \mathcal{G}$ which is a topologically trivial flat $T$-gerbe. The field in toral Chern-Simons is a 'non-flat trivialization' $P$ of $\exp \partial \mathcal{G}$. More precisely, there is a groupoid whose objects are $\mathfrak{t}$ gerbes on $Y$ and whose morphisms are torsors for the differential cohomology group $\widetilde{H \Pi}^{2}(Y)$; these morphisms are equivalence classes of non-flat trivializations of the exponentials of the $\mathfrak{t}$-gerbes. (The actual non-flat trivializations are morphisms in a 2-groupoid.) Now $P$ extends to a non-flat trivialization of $\exp \mathcal{G}$ on $X$. Let $\omega \in \Omega^{2}(X ; \mathfrak{t})$ be its curvature. Work over a base manifold $S$. Then the quadratic form (5.21), applied to $\partial \mathcal{G}$ and integrated over the fibers of $Y \rightarrow S$, yields a flat $\mathbb{R} / \mathbb{Z}$ bundle over $S$ whose equivalence class in $H^{1}(S ; \mathbb{R} / \mathbb{Z})$ is computed by a relative version of (5.22) for the relative 3 -manifold $Y \rightarrow S$. Because $\partial \mathcal{G}$ is extended to a $\mathfrak{t}$-gerbe on $X$, this $\mathbb{R} / \mathbb{Z}$-bundle comes with a trivialization. The Chern-Simons action is a section of this circle bundle, and using the trivialization may be identified with the function

$$
\int_{X / Z}\langle\omega \wedge \omega\rangle \quad(\bmod \mathbb{Z})
$$

on $S$.

In the quantum Chern-Simons theory we integrate the exponential of (5.24) over the stack of non-flat trivializations $P$ for fixed $\mathcal{G}$. The result lives in the complex line bundle $L(\partial \mathcal{G}) \rightarrow S$ which is the exponential of the $\mathbb{R} / \mathbb{Z}$-bundle in the previous paragraph. Automorphisms of $\partial \mathcal{G}$ act on $L(\partial \mathcal{G})$ and the result of the path integral is invariant. Therefore, if these automorphism act nontrivially the path integral vanishes. (This is called the 'Gauss law' in physics.) Now an automorphism $\alpha$ of $\partial \mathcal{G}$ acts through its equivalence class $[\alpha] \in H^{1}(Y ; \mathfrak{t})$, and the action only depends on the equivalence class $[\partial \mathcal{G}] \in H^{2}(Y ; \mathfrak{t})$ of the gerbe: namely, it acts as multiplication by $\exp (2 \pi i\langle[\alpha] \smile[\partial \mathcal{G}]\rangle)$. This shows that the path integral vanishes unless $\partial \mathcal{G}$ is trivializable as a flat $\mathfrak{t}$-gerbe. Relative to a trivialization the Chern-Simons field $P$ is a usual $T$-bundle with connection and we recover the standard description of classical Chern-Simons.

\section{The basic tensor category and its center}

\section{$\S 6.1$. Drinfeld centers}

Let $T$ be a torus, $\tau \in H^{4}(B T ; \mathbb{Z})$ a non-degenerate twisting. Recall from Proposition 5.16 the line bundle $K \rightarrow T \times T$, which is a 2-cocycle. Define a convolution on the category Sky[T] of sky-scraper sheaves of finite-dimensional vector spaces on $T$, with finite support, by setting

$$
\mathbb{C}_{x} * \mathbb{C}_{y}=K_{x, y} \otimes \mathbb{C}_{x y}
$$

for the skyscrapers at $x, y, x y \in T$. The cocycle property of $K$ ensures that this defines a tensor category, which we denote by $\operatorname{Sky}^{\tau}[T]$. This is an analogue of the "twisted group $\operatorname{ring}^{\prime} \operatorname{Vect}^{\tau}[G]$ discussed in $\S 4.2$ for finite groups $G$. 
The tensor structure lifts to the universal cover $\mathfrak{t}$ of $T$, but it is trivializable there. This is because we can trivialize the pullback of $K$ as a 2-cocycle valued in Pic, compatibly with our chosen trivialization of the pullback of $L$, as in (5.14) and (5.5). However, we shall see that $\mathrm{Sky}^{\tau}[\mathfrak{t}]$ carries a higher structure, a braiding. This is specified by a family of automorphism $\mathbb{C}_{\xi} * \mathbb{C}_{\xi^{\prime}} \stackrel{\sim}{\longrightarrow}$ $\mathbb{C}_{\xi^{\prime}} * \mathbb{C}_{\xi}, \xi, \xi^{\prime} \in \mathfrak{t}$ forming a bi-multiplicative section of the line bundle $L$ of (5.5). In the defining trivialization, the distinguished section is the function

$$
\sigma\left(\xi, \xi^{\prime}\right)=\exp \left\{-\pi i\left\langle\xi, \xi^{\prime}\right\rangle\right\}
$$

The structure extends in fact to the larger category $\mathrm{Sky}^{\tau}[C]$ of sky-scrapers on the correspondence space $C=(\mathfrak{t} \times \Lambda) / \Pi$ in (5.7) by a reformulation of Proposition 5.9(ii). Alternatively, there is a natural quadratic function $\theta: C \rightarrow \mathbb{T}$ (Remark 6.2(ii) below), which determines the braided tensor structure on $\mathrm{Sky}^{\tau}[C]$ by a general construction (cf. the end of $\S 8.1$ ). The conceptual meaning for this structure is given by the following.

Proposition 6.1. (i) The braided tensor category $\mathrm{Sky}^{\tau}[C]$ is the "continuous" Drinfeld center of $\mathrm{Sky}^{\tau}[T]$. The natural functor from $\mathrm{Sky}^{\tau}[C]$ to $\mathrm{Sky}^{\tau}[T]$ is induced by projection.

(ii) As braided tensor categories, $\operatorname{Sky}^{\tau}[C] \equiv \operatorname{Sky}^{\tau}[\mathfrak{t}] \otimes \operatorname{Sky}^{\tau}[\hat{F}]$, sitting in $C$ by the obvious inclusions, and lifting the splitting $C=\mathfrak{t} \times \hat{F}$ of abelian groups. Moreover, the two factors are mutual commutants.

The last statement means that any $\operatorname{Sky}^{\tau}[C]$-object braiding trivially with all of $\operatorname{Sky}^{\tau}[\hat{F}]$ is in $\mathrm{Sky}^{\tau}[\mathfrak{t}]$, and similarly with $\hat{F}$ and $\mathfrak{t}$ interchanged.

Regarding the notion of "continuous Drinfeld center", we will limit ourselves to the following observation. All our categories are semi-simple, with the simple isomorphism classes corresponding to the points on the underlying spaces. They are 'categorifications' of the underlying abelian groups. We then ask that the half-braiding in Definition 4.8 should be continuous on irreducible objects, in their natural topology. Developing this in more detail would be a distraction here for two reasons: first, the key ingredient for us is not quite the Drinfeld center, which is a Hochschild cohomology, but its dual notion, a Hochschild homology, which is a tensor product. Second, as we briefly indicate in the next section, the easiest way to justify our story rigorously is via approximations by finite abelian groups, in the time-tested "lattice approximation" of quantum field theory; and continuity plays no rôle in that setting.

Sketch of proof. An alternative description of $C$ will be useful. Note that $\tau$ defines an isogeny $\tau_{\mathbb{T}}: T \rightarrow T^{*}$, the Langlands dual torus. Its kernel is the group $F$ described in Notation 5.13 . Interpreting $T^{*}$ as the moduli space of flat line bundles on $T$, the map $\tau_{\mathbb{T}}$ classifies the line bundle L. Then,

$$
C \cong \mathfrak{t}^{*} \underset{T^{*}}{\times} T .
$$

To identify this fiber product over $T^{*}$ with the standard description $(\mathfrak{t} \oplus \Lambda) / \Pi$, send $(\xi, \lambda)$ in the latter space to $\left(d \tau(\xi)-\lambda, e^{\xi}\right)$ in the former.

In the second description of $C$, we interpret a point $x \in T$ as the sky-scraper object $\mathbb{C}_{x}$ and an element in the fiber of $\tau_{\mathbb{T}}(x)$ as a continuous character of the central extension of the group $T$ 
defined by $L_{x,-}$; see Proposition 5.9(ii). But this is precisely a continuous half-braiding of $\mathbb{C}_{x}$ with the simple objects of $\mathrm{Sky}^{\tau}[T]$. It is easy to see a priori that simple objects of the center must be supported at single points, and then that they have rank one, so we have just found all of them.

If we start at the identity with the trivial braiding, and keep a continuous choice of character as we move in $\mathfrak{t}$, we sweep out the identity copy of $\mathfrak{t}$ in $C$. The projective characters of $T$ thus swept out have the property that they are trivial on $F \subset T$ (where every central extension in the family is naturally trivialized). On the other hand, $L_{x,-}$ has trivial holonomy at the points $x \in F$, and there we can choose the trivial character in $\mathfrak{t}^{*}$. This defines the copy $\hat{F}$ of $F$ in $C$. Clearly, this braiding commutes with the braiding by the copy of $\mathfrak{t}$ just described.

Non-degeneracy of the quadratic form on $\mathfrak{t}$ implies that its commutant is no larger than $\mathrm{Sky}^{\tau}[F]$. That the two in fact commute, and the analogous statement for $F$, follow from the formula for the ribbon element below. One can also argue directly that the braiding is defined by the perfect bi-character on $C$ described in Remark 6.2.

Remark 6.2. (i) The two descriptions of $C$ make clear its remarkable property of being Pontrjagin self-dual: the groups $\mathfrak{t}^{*} \times T$ and $\mathfrak{t} \times \Lambda$ are Pontrjagin dual, and the kernel of the addition map of the first group to $T^{*}$ is dual to the quotient of the second by the dual inclusion of $\Pi$. This self-duality is symmetric, and is induced by the quadratic "ribbon" map $\theta: C \rightarrow \mathbb{T}$ below.

(ii) The braided tensor category $\operatorname{Sky}^{\tau}[C]$ and its sub-categories $\operatorname{Sky}^{\tau}[\hat{F}], \operatorname{Sky}^{\tau}[\mathfrak{t}]$ are in fact ribbon categories with ribbon function on the simple object $(\xi, \lambda)$

$$
\theta(\xi, \lambda)=\exp \pi i\left\{\left\|\tau^{-1}(\lambda)\right\|^{2}-\left\|\xi-\tau^{-1}(\lambda)\right\|^{2}\right\}
$$

using the norm associated to the quadratic form (5.2). The square of the braiding is given by the standard formula $\theta(X Y) \theta^{-1}(X) \theta^{-1}(Y)$.

(iii) The equivalence in 6.1(ii) is not one of ribbon categories, because the ribbon function is quadratic and not linear on objects.

(iv) One should mind that the tensor structure defined by the restriction $\tau \in H^{4}(B F ; \mathbb{Z})$ is not necessarily trivial, that is, $\mathrm{Sky}^{\tau}[F]$ may differ from $\mathrm{Sky}[F]$ as a tensor category. For instance, this always happens when $T=S^{1}$. But $\tau$ is always 2-torsion on $F$.

(v) The category $\mathrm{Sky}^{\tau}[\hat{F}]$ is in fact a modular tensor category [BK, T] and defines the 1-2-3dimensional Chern-Simons theory (of framed manifolds) associated to the torus $T$ at level $\tau$ via the Reshetikhin-Turaev theorem.

\section{$\S 6.2$. Finite approximation of $T$ and $\mathfrak{t}$}

Let us now describe the finite ("lattice") approximations of $C, \mathfrak{t}, T$ and develop the finite version of Chern-Simons theory in the next section. Let $n$ be positive integer, which will become infinitely divisible in the limit13; denote by $T_{(n)}^{*} \subset T^{*}$ the subgroup of $n$-torsion points and by $T^{(n)} \rightarrow T$ the dual covering torus of $T$, with Galois group $\Pi^{(n)} \cong \Pi / n \Pi$, Pontrjagin dual to $T_{(n)}^{*}$. Finally, let $T_{(n F)}$ denote the inverse image of $T_{(n)}^{*}$ in $T$ under $\tau_{\mathbb{T}}$ and $\mathfrak{t}^{(n)}$ that of $T_{(n F)}$ in $T^{(n)}$. There is also the Pontrjagin dual $\mathfrak{t}^{*(n F)}$ of $\mathfrak{t}^{(n)}$, which is a Galois cover of $T_{(n)}^{*}\left(\right.$ restricted from $\left.T^{*}\right)$ with group

\footnotetext{
${ }^{13}$ That is, we'll take the limit over $\mathbb{N}$ ordered by divisibility.
} 
$\Lambda^{(n F)}$, Pontrjagin dual to $T_{(n F)}$. It is an exercise to check that the restriction $\tau_{\mathbb{T}}: T_{(n F)} \rightarrow T_{(n)}^{*}$ lifts naturally to an isomorphism $\mathfrak{t}^{(n)} \cong \mathfrak{t}^{*(n F)}$.

As $n \rightarrow \infty$, the finite group $T_{(n)}$ will be our approximation for $T, \mathfrak{t}^{(n)}$ will approximate the Lie algebra, $\mathfrak{t}^{*(n F)}$ its dual, $T_{(n)}^{*}$ will play the rôle of $T^{*}, \Pi^{(n)}$ that of $\Pi$ and $\Lambda^{(n F)}$ that of $\Lambda$.

Remark 6.3. If $d$ is the dimension of $T$, then $\# T_{(n)}^{*}=n^{d}, \# T_{(n F)}=\# F \cdot n^{d}, \# \mathfrak{t}^{(n)}=\# F \cdot n^{2 d}$.

The twisting $\tau$ restricts to $H^{4}\left(B T_{(n)} ; \mathbb{Z}\right)$ and defines a tensor category $\operatorname{Vect}^{\tau}\left(T_{(n)}\right)$ as discussed in $§ 4.2$, which is a full subcategory of $\operatorname{Sky}^{\tau}[T]$. Consider

$$
C^{(n)}:=\left(\mathfrak{t}^{(n)} \oplus \Lambda^{(n F)}\right) / \Pi^{(n)} \cong \mathfrak{t}^{*(n F)} \underset{T_{(n)}^{*}}{\times} T_{(n F)}
$$

It is easy to check that the ribbon function $\theta$ of Remark 6.2(ii) descends to a non-degenerate quadratic function on $C^{(n)}$, and gives a braided tensor structure on $\mathrm{Sky}^{\tau}\left[C^{(n]}\right)$, with the restricted twisting $\tau$ ). The projection $\mathfrak{t}^{*(n F)} \cong \mathfrak{t}^{(n)} \rightarrow T_{(n)}$ defines a splitting $C^{(n)} \cong \mathfrak{t}^{(n)} \times \hat{F}$. The proof of the following, discrete analogue of Proposition 6.1 is left to the reader.

Proposition 6.4. (i) The braided tensor category $\mathrm{Sky}^{\tau}\left[C^{(n)}\right]$ is the Drinfeld center of $\mathrm{Sky}^{\tau}\left[T_{(n F)}\right]$. The natural functor from $\mathrm{Sky}^{\tau}\left[C^{(n)}\right]$ to $\mathrm{Sky}^{\tau}\left[T_{(n)}\right]$ is induced by projection.

(ii) As braided tensor categories, $\mathrm{Sky}^{\tau}\left[C^{(n)}\right] \equiv \operatorname{Sky}^{\tau}\left[\mathfrak{t}^{(n)}\right] \otimes \operatorname{Sky}^{\tau}[\hat{F}]$, sitting in $C^{(n)}$ by the obvious inclusions, and lifting the splitting $C^{(n)}=\mathfrak{t}^{(n)} \times F^{*}$ of abelian groups. Moreover, the two factors are mutual commutants.

\section{$\S 6.3$. Morita relations between our categories}

The categories discussed in this section are closely related. Just as the right notion of quasiisomorphism for algebras in their natural world is Morita equivalence, there is a corresponding notion for tensor categories and even braided tensor categories. In the next section, we sketch a minimal background for these notions; but let us now state a key result and its significance for TQFTs.

Proposition 6.5. (i) $\operatorname{Sky}^{\tau}[\mathfrak{t}]$ and $\operatorname{Sky}^{\tau}[\hat{F}]$ are Morita equivalent by means of $\operatorname{Sky}^{\tau}[T]$.

(ii) $\mathrm{Sky}^{\tau}\left[\mathfrak{t}^{(n]}\right)$ and $\mathrm{Sky}^{\tau}[\hat{F}]$ are Morita equivalent by means of $\mathrm{Sky}^{\tau}\left[T_{(n)}\right]$.

(iii) All these BTCs are quasi-invertible, more precisely, $\operatorname{Sky}^{\tau}[S] \otimes \mathrm{Sky}^{-\tau}[S]$ is Morita equivalent to Vect in all cases $S=\mathfrak{t}, \hat{F}, \mathfrak{t}^{(n)}$ (with $(-\tau)$ indicating the opposite braiding).

Thus, $\mathrm{Sky}^{\tau}[\mathfrak{t}]$ and $\mathrm{Sky}^{\tau}\left[\mathfrak{t}^{(n)}\right]$ are quasi-invertible in their world. Moreover, they are equivalent to the unit braided tensor category Vect in the following cases:

- When the signature of $\langle-,-\rangle$ on $\mathfrak{t}$ is divisible by 8 ;

- When $\mathbb{Z} / 2$-graded vector spaces are used.

In the first case, we apply Proposition 6.5(i) to a product $T$ of copies of the $E_{8}$ maximal torus, with its generating class $\tau$; unimodularity of the $E_{8}$ lattice ensures that $F=\{1\}$. The second case uses a variant of our categories and twistings with graded vector spaces, which we have not discussed. In this case, with $T=\mathrm{U}(1)$, the graded tensor category $\mathrm{Gr}-\operatorname{Vect}(\mathrm{U}(1))$ can be twisted by a class 
which is half 14 the generator of $H^{4}(B U(1) ; \mathbb{Z})$, and leads again to a trivial group $F$. (The group $F$ corresponding to the generator of $H^{4}(B \mathrm{U}(1) ; \mathbb{Z})$ has two elements.)

The braided tensor categories above lead to invertible 4-dimensional TQFTs for oriented manifolds: these are the theories described in $\$ 5.2$. Invertibility has the obvious meaning, in the tensor structure on TQFTs, and follows from Proposition 6.5(iii). Graded vector spaces, however, require spin structures on the manifold. We conclude that the (isomorphic) theories defined by $\mathrm{Sky}^{\tau}[\mathfrak{t}]$ and $\mathrm{Sky}^{\tau}\left[\mathfrak{t}^{(n)}\right]$ are trivial on oriented manifolds when the signature of $\tau$ is a multiple of 8 , and always trivial on spin manifolds 15

\section{Higher algebra: tensor and bi-module categories}

\section{§7.1. $m$-Algebras}

Our picture of Chern-Simons theory requires an ascent to categorical altitudes which exceed the safe limit without special equipment: we will need linear 3-categories describing (very simple) 4dimensional theories. Fortunately, there is a recursive procedure to produce the higher categorical objects needed and, even more fortunately, up to the range of dimensions we study there are user-friendly models for these. We hope to return to an extensive discussion of these structures elsewhere; here we sketch their basic features.

Recall first that vector spaces and linear maps form a symmetric tensor category, and that algebra objects in a symmetric tensor category form in turn a symmetric tensor category (of one level higher, but who's counting?).

Definition 7.1. A 0-algebra is a complex vector space, and a morphism of 0-algebras is a linear map. For $m>0$, an $m$-algebra is an algebra object in the (symmetric tensor $m$-) category of $(m-1)$ algebras. Morphism between m-algebras are bi-module objects in the category of $(m-1)$-algebras. (Higher morphisms are defined recursively.)

The 2-category of (1-)algebras, modules and intertwiners is familiar enough - see 2.13 - and here we just give analogous pictures (or adequate substitutes) for the next two levels. A 2-algebra is an algebra $A$ together with an $\left(A, A^{\otimes 2}\right)$-bi-module $M$ defining the 2-multiplication and a left $A$-module $E$ defining the identity; there must be the associativity and unit intertwiners (2-morphisms in the category of algebras) satisfying the 'obvious' compatibility rules. A morphism of 2-algebras, from $A$ to $B$, is a $(B, A)$ bi-module in the world of algebras: an algebra $N$, plus an $(N, B \otimes N)$-bi-module $P$ and an $(N, N \otimes A)$-bi-module $Q$, plus compatibility isomorphisms defining the bi-algebra structure.

In general, a 2-algebra structure on $A$ defines a tensor structure on the category of left $A$-modules; sending $A \mapsto(A-\mathrm{Mod})$ is a fully faithful functor from the 3-category of 2-algebras to that of tensor

\footnotetext{
${ }^{14}$ This half-generator is defined in the generalized cohomology theory $h$ of Remark 1.3 this leads to a TQFT for spin, rather than oriented manifolds.

${ }^{15}$ Some structure has been swept under the carpet here. Defining the theory for oriented or spin manifolds, rather than framed ones, requires us to specify an action of $\mathrm{SO}(4)$ or $\mathrm{Spin}(4)$, which adds some parameters. One of those is the constant $\lambda$ coupled to the Euler class in (5.23).
} 
categories. Modulo the problem of realizing linear categories as module categories, one can use tensor categories instead of 2-algebras. This, of course, is what we have been doing.

For example, any commutative algebra becomes a 2-algebra via its own multiplication map; a morphism between commutative algebras $A$ and $B$, viewed as 2-algebras, is simply a $B \otimes A$ algebra. However, commutative algebras have a more obvious and economical embedding into tensor categories, sending $A$ to the tensor category with one object and endomorphism ring $A$. We will write $A[2]$ when thinking of $A$ as a 2-algebra.

More relevant is the example of a Hopf algebra $H$, which becomes a 2-algebra by means of the multiplication $M:=H \otimes H$, which is a left $H$-module via the Hopf structure. For the Hopf algebra $A=\mathbb{C}(G)$ of a finite group with the point-wise multiplication, but co-multiplication induced by the Hopf structure, the category ( $A$-Mod) is that of vector bundles over $G$; and the tensor category defined by the Hopf 2-algebra structure is precisely the earlier Vect $[G]$ in $\S 4.2$. A twisting class $\tau \in H^{4}(B G ; \mathbb{Z})$ changes the associator of the 2-multiplication.

One level up, a 3-algebra structure on $A$ can be interpreted as follows: the 2-algebra structure turns $(A$-Mod) into a tensor category, having a 2-category of modules; and the 3 -algebra structure gives a tensor structure on the latter 2-category. (This, in turn, has a linear 3-category of modules, affirming the 3-categorical nature of a 3 -algebra.) For example, if $A$ is commutative, two ( $A$-Mod)modules can be tensored over $(A-\mathrm{Mod})$ to produce a new $(A-\mathrm{Mod})$-module, since the latter is a symmetric tensor category. Similarly, in the case of the group ring, Vect $[G]$-modules can be tensored over Vect $[G]$, using the Hopf structure on the latter. This is usually not possible for the twisted versions $\operatorname{Vect}^{\tau}[G]$, where the Hopf structure is broken by the twisting; this is much like the case of twisted group rings over $\mathbb{C}$ defined from $\mathbb{T}$-central extensions of the group, which do not usually have 2-algebra promotions.

\section{§7.2. Braided tensor categories}

Manipulating tensor 2-categories can be rather daunting, and no doubt the most general 3algebras are no friendlier; but in some cases they can be captured by a more concise structure, namely a braided tensor category (BTC). These are special kinds of algebra objects in the category of all tensor categories, and represent the simplest type of structure on the category $(A-$ Mod) which promotes $A$ to a 3 -algebra. For example, each braided tensor category $\operatorname{Sky}^{\tau}[S]$ of the previous section promotes the algebra of functions on $S$, with pointwise multiplication, into a 3-algebra 16 As we will see, these higher structures are the natural result of quantization.

Even if we restrict to BTC among all algebra objects in the symmetric 3-category of tensor categories, the description of all module objects - hence the description of bi-module objects, which are the morphisms in the 4-category of BTCs - can be awkward; see the case of 2-algebras above. However, there is a nice class of special module objects $M$, those for which the action of $B$ on $M$ is defined by a functor $B \otimes M \rightarrow M$. This must be a tensor functor, which forces the action of $B$ to half-braid with the multiplication on $M$; in other words, the algebra map $B \rightarrow M$ induced by tensoring with $1 \in M$ must lift to a braided tensor functor into the Drinfeld center $Z(M)$. We call these ' $B$-modules' half-braided algebras over $B$. The reader should think of the analogy with a

\footnotetext{
${ }^{16}$ The cases of $S=\mathfrak{t}$ and $S=C$ require a priori a grain of salt, because we are considering very special modules, but in fact the structure supplied does give a 3 -algebra in each case, albeit with poor finiteness conditions.
} 
commutative 1-algebra $A$ and the 2-category of central algebras over it; here, the braiding can be thought of as a homotopy between left and right multiplications.

In spite of these difficulties, the following simplified four-category $\mathcal{C}$ will be a suitable replacement the 4-category of 3-algebras. Objects of $\mathcal{C}$ will be braided tensor categories; morphisms from $A$ to $B$ will be half-braided $(B, A)$ bi-algebra categories, that is, tensor categories $M$ with a braided tensor functor $B \otimes A^{o p}$ to $Z(M)$. 2-morphisms will be bi-modules between bi-algebras (with compatible $B \otimes A^{o p}$-action), and then functors and natural transformations round this up.

\section{§7.3. Quasi-isomorphisms}

The key moral of the story is that the correct notion of equivalence becomes increasingly obscure for higher algebras, although not less precise. The correct notion is always a pair of functors $f, g$ with $f \circ g$ and $g \circ f$ both equivalent to the respective identities 17 Thus, for 1-algebras we have Morita equivalence. For 2-algebras, the familiar Morita conditions

$$
P \underset{R}{\otimes} Q \equiv S, \quad Q \underset{S}{\otimes} P \equiv R
$$

become Morita equivalences themselves, as $P$ and $Q$ are now bi-algebras. This continues, as we now illustrate in the proof of Proposition 6.5. Recall for this purpose the braided tensor categories $\operatorname{Sky}^{\tau}[\hat{F}], \operatorname{Sky}^{\tau}[\mathfrak{t}], \mathrm{Sky}^{\tau}[C]$ from the previous section, the latter two accompanied by their finite approximations $\operatorname{Sky}^{\tau}\left[\mathfrak{t}^{(n)}\right]$ and $\mathrm{Sky}^{\tau}\left[C^{(n)}\right]$. In addition, the tensor categories $\mathrm{Sky}^{\tau}[T]$ and $\operatorname{Sky}^{\tau}\left[T_{(n)}\right]$ are half-braided bi-algebras over the first three, respectively their finite versions. This makes them into 1 -morphisms in the 4-category $\mathcal{C}$.

Sketch of proof of Proposition 6.5. We prove part (ii), part (i) is similar. We must produce Morita equivalences

$$
\begin{aligned}
& \operatorname{Sky}^{\tau}\left[T_{(n)}\right] \underset{\mathrm{Sky}^{\tau}\left[\mathfrak{t}^{(n)}\right]}{\bigotimes} \operatorname{Sky}^{\tau}\left[T_{(n)}\right] \sim \operatorname{Sky}^{\tau}[\hat{F}], \\
& \operatorname{Sky}^{\tau}\left[T_{(n)}\right] \underset{\operatorname{Sky}^{\tau}[\hat{F}]}{\bigotimes} \operatorname{Sky}^{\tau}\left[T_{(n)}\right] \sim \operatorname{Sky}^{\tau}\left[\mathfrak{t}^{(n)}\right],
\end{aligned}
$$

and similarly for $\mathfrak{t}$ and $T$. The Morita objects realizing (17.2) are $\operatorname{Sky}^{\tau}\left[T_{(n)}\right]$ in all cases (respectively, $\mathrm{Sky}^{\tau}[T]$ for part (i)). Indeed, the desired identity is

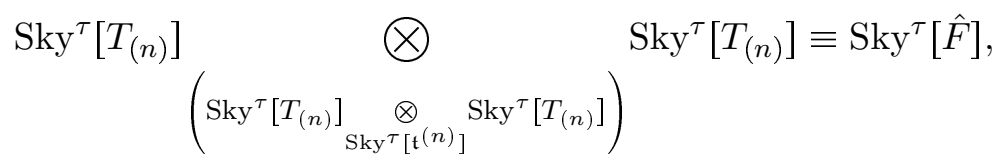

and the obvious permutations. Were we to tensor over the braided tensor category $\operatorname{Sky}^{\tau}\left[T_{(n)}\right] \otimes$ $\mathrm{Sky}^{\tau}\left[T_{(n)}\right]$ instead, the left side would be the Hochschild homology $A \underset{A \otimes A}{\otimes} A$. By semi-simplicity (or rather, self-duality of $\mathrm{Sky}^{\tau}\left[T_{(n)}\right]$ as a self-bi-module), this would give (a linear category equivalent

\footnotetext{
${ }^{17}$ This is not circular, since it relies on the lower-algebra definition of equivalence.
} 
to) the Drinfeld center $\operatorname{Sky}^{\tau}\left[C^{(n)}\right]$ of $\operatorname{Sky}^{\tau}\left[T_{(n)}\right]$. Instead, the effect of working over the tensor product

$$
\operatorname{Sky}^{\tau}\left[T_{(n)}\right] \underset{\operatorname{Sky}^{\tau}\left[\mathfrak{t}^{(n)}\right]}{\otimes} \operatorname{Sky}^{\tau}\left[T_{(n)}\right]
$$

is to pick the relative Drinfeld center of $\operatorname{Sky}^{\tau}\left[T_{(n)}\right]$ over $\operatorname{Sky}^{\tau}\left[\mathfrak{t}^{(n)}\right]$, the commutant of $\operatorname{Sky}^{\tau}\left[\mathfrak{t}^{(n)}\right]$ in the full center $\mathrm{Sky}^{\tau}\left[C^{(n)}\right]$. By Proposition 6.4, this is $\operatorname{Sky}^{\tau}[\hat{F}]$. Exchanging $\hat{F}$ and $\mathfrak{t}^{n}$ and repeating the argument we obtain the second identity in (7.2).

Regarding Part (iii), this is a similar argument. Noting that $\mathrm{Sky}^{-\tau}[S]$ can be identified with the opposite braided tensor category of $\mathrm{Sky}^{\tau}[S]$, we will use the tensor category $\mathrm{Sky}^{\tau}[S]$ with its left-right action on itself as Morita bi-module. Furthermore, we use $\operatorname{Sky}^{\tau}[S]$ again to produce the Morita equivalence of

$$
\mathrm{Sky}^{\tau}[S] \bigotimes_{\mathrm{Sky}^{\tau}[S] \otimes \mathrm{Sky}^{-\tau}[S]} \mathrm{Sky}^{\tau}[S]
$$

with Vect. Indeed, after identifying one copy of $\mathrm{Sky}^{\tau}[S]$ with the dual category, the tensor square of $\mathrm{Sky}^{\tau}[S]$ over the left category is the relative Drinfeld center of $\mathrm{Sky}^{\tau}[S]$ over $\mathrm{Sky}^{\tau}[S] \otimes \mathrm{Sky}^{-\tau}[S]$. However, the center is the double $\operatorname{Sky}^{\tau}[S] \otimes \operatorname{Sky}^{\tau}\left[S^{*}\right]$, and because $\tau$ gives an isomorphism $S \cong S^{*}$, this double is nothing but $\mathrm{Sky}^{\tau}[S] \otimes \mathrm{Sky}^{-\tau}[S]$ again, with its half-braided tensor action on $\mathrm{Sky}^{\tau}[S]$. Non-degeneracy of $\tau$ again ensures that the identity is the only simple object braiding trivially with everything; therefore the relative center is Vect, as desired.

\section{Quantization of groupoids}

In this section, we outline a "canonical quantization" procedure starting from a "classical" topological quantum field theories with target higher groupoids (satisfying some finiteness conditions). In other words, we elaborate on the map $\mathrm{Sum}_{n}$ in (3.9). For our purposes, "higher groupoids" are spaces, with 0-groupoids being discrete sets and ordinary, or 1-groupoids being homotopy 1 -types. The case $n=2$ is used in $\S 5.2$. The constraint is finiteness of the homotopy groups. We hope to develop the full story elsewhere; here we outline the construction while flagging the cases of immediate interest.

There is a quantization procedure for each $n \geqslant 0$, leading to a TQFT in the respective dimension. At its root is a linearization functor from spaces to higher algebras. (This works without finiteness.) This "higher groupoid ring" is an $m$-algebra, which is what a TQFT in dimension $n=m+1$ assigns to a point. The construction enhances the group algebra of a finite group $(m=1)$, or even more basically, of the vector space of functions on a finite set $(m=0)$. The discrepancy between $m$ (the algebra level) and $n$ (the TQFT dimension) - for which we apologize - is caused by the fact that the term $n$-vector space has been used for a much more restrictive notion than our $(n-1)$-algebras.

The $m$-algebra associated to a (connected, pointed) space $X$ has as its category of modules the $m$-category of representations of the based loop space $\Omega X$ on $(m-1)$-algebras; for disconnected spaces, we must sum over components. The dependence on base points can be removed by viewing 
its representations as the category of local systems of $(m-1)$-algebras over $X$. We will write $R_{m}(X)$ for the groupoid algebra when not relying on base-point choices.

There is an untwisted construction, leading to a TQFT of unoriented manifolds, and a construction with twists by a phase, which requires orientations.

Notation 8.1. Let $G$ be a group and $A$ an algebra. Then $A[G]$ denotes the group algebra of $G$ under convolution and $A(G)$ the $A$-valued functions on $G$ under pointwise multiplication.

\section{$\S 8.1$. Low $m$ examples}

Let $\mathrm{X}$ be a space, which for our application may be assumed to have finitely many nonzero homotopy groups, each of which is finite. We now produce a candidate for the $m$-algebra $R_{m}(X)$.

For $m=-1$, our construction produces the number of isomorphism classes of points of the groupoid, weighted by their automorphisms;

$$
\sum_{x \in \pi_{0} X} \prod_{i \geqslant 1}\left(\# \pi_{i}(X, x)\right)^{(-1)^{i}}
$$

A number counts as a "(-1)-algebra"; for natural numbers at least, which are cardinalities of sets, this matches our intuition. The weight of each isomorphism class is the alternating product of the orders of the homotopy groups, which places an obvious finiteness condition. Without it, we must abandon the top layer of the TQFT.

For $m=0$, we get the vector space of functions on $\pi_{0} X$.

For $m=1$, we produce the usual groupoid algebra. After a choice of base-points, this is the direct sum of the group algebras of the $\pi_{1}$ 's of the components, but a Morita equivalent base-point free construction is the path algebra of $X 18$

Things become more interesting for $m=2$ : the groupoid $X=K\left(\pi_{2}, 2\right)$ quantizes to the 2algebra $\mathbb{C}\left[\pi_{2}\right][2]$ associated to the commutative group algebra $\mathbb{C}\left[\pi_{2}\right]$. If, in addition, a $\pi_{1}$ is present, we get a crossed product 2-algebra $\pi_{1} \ltimes \mathbb{C}\left[\pi_{2}\right][2]$. This easiest to describe by means of the tensor structure on the category of modules of the underlying 1-algebra. As a 1-algebra, $\pi_{1} \ltimes \mathbb{C}\left[\pi_{2}\right][2]$ consists of the functions on $\pi_{1}$ with values in the algebra $\mathbb{C}\left[\pi_{2}\right]$, and point-wise multiplication. Its linear category of modules $\operatorname{Rep}\left(\pi_{2}\right)\left(\pi_{1}\right)$, consisting of bundles of $\pi_{2}$-representations over $\pi_{1}$. Now, the tensor category of $\mathbb{C}\left[\pi_{2}\right][2]$-modules is equivalent to $\operatorname{Rep}\left(\pi_{2}\right)$ as a linear category, but carries the non-standard tensor structure corresponding to convolution of characters. In the Fourier transformed picture, this is the category $\operatorname{Vect}\left(\pi_{2}^{*}\right)$ of vector bundles on the Pontryagin dual group, but with the point-wise tensor structure 19 The group $\pi_{1}$ acts by automorphisms of the tensor category $\operatorname{Vect}\left(\pi_{2}^{*}\right)$ : the action comprises the obvious automorphisms of $\pi_{2}$, as well as the $k$-invariant $k \in H^{3}\left(B \pi_{1} ; \pi_{2}\right)$, if present. Indeed, we may interpret $k$ as a crossed homomorphism $\pi_{1} \rightarrow B^{2} \pi_{2}$, and $\pi_{2}$ is a group of central 2-automorphisms of $\operatorname{Vect}\left(\pi_{2}^{*}\right)$ as a tensor category: namely, elements of $\pi_{2}$ give 1-automorphisms (multiplications by Fourier modes) of $\operatorname{Vect}\left(\pi_{2}^{*}\right)$ as a bi-module category over itself, which as such represents the identity morphism on the tensor category $\operatorname{Vect}\left(\pi_{2}^{*}\right)$. Thus,

\footnotetext{
${ }^{18}$ We are assuming a discrete model for spaces, such as simplicial sets.

${ }^{19}$ The standard tensor structure on $\operatorname{Rep}\left(\pi_{2}\right)$ would correspond to convolution on $\operatorname{Vect}\left(\pi_{2}^{*}\right)$.
} 
we can form the desired crossed-product tensor category $20 \pi_{1} \ltimes \operatorname{Vect}\left(\pi_{2}^{*}\right)$ of modules for $\pi_{1} \ltimes \mathbb{C}\left[\pi_{2}\right][2]$. More succinctly but loosely, when $\pi_{1}$ acts trivially and the $k$-invariant is null, $H=\mathbb{C}\left[\pi_{2}\right]\left(\pi_{1}\right)$ is a Hopf algebra over $\mathbb{C}\left[\pi_{2}\right]$ and this gives a 2-algebra structure as explained in $\$ 7.1$. A non-trivial action of $\pi_{1}$ is incorporated by twisting the left $H$-module structure over $H$, as is the $k$-invariant.

The case $m=3$ gives rise to 3-algebras. Each connected component $X_{x}$ is $B \Omega_{x} X$, and $R_{3}\left(X_{x}\right)$ is the promotion of $R_{2}\left(\Omega_{x} X\right)$ to a 3-algebra using the extra multiplication in the loop space. However, for simply connected $X$, a braided tensor category arises naturally. Namely, the tensor category $\pi_{2} \ltimes_{\Omega k} \operatorname{Vect}\left(\pi_{3}^{*}\right)$ was defined from the second loop space $\Omega^{2} X$, which has a homotopycommutative multiplication, homotopy-commuting with the third multiplication on $R_{3}\left(X_{x}\right)$. (The action of $\pi_{2}$ is classified by the looping $\Omega k \in H^{3}\left(B \pi_{2} ; \pi_{3}\right)$ of the unique Postnikov invariant of $X$, $k \in H^{4}\left(K\left(\pi_{2} ; 2\right) ; \pi_{3}\right)$.) Otherwise put, the category $\pi_{2} \ltimes_{\Omega k} \operatorname{Vect}\left(\pi_{3}^{*}\right)$ has a second multiplication compatible with the first: this structure is equivalent to a braiding. A $\pi_{1}$, of course, would spoil the requisite commutativity.

Remark 8.3. Recall that $k$ is equivalent to the datum of a quadratic map $\pi_{2} \rightarrow \pi_{3}$ (Lemma 5.17), so this quadratic map is all that is needed for the construction of the braiding on the category $\pi_{2} \ltimes_{\Omega k} \operatorname{Rep}\left(\pi_{3}\right)$. When $\pi_{3}$ is a subgroup of $\mathbb{T}, B^{2} \pi_{3}$ acts by automorphisms of the 2-algebra $\mathbb{C}[2]$ (equivalently, automorphisms of the tensor category Vect), and we see from the same construction that a braiding on Vect $\left[\pi_{2}\right]$ is determined by a $\mathbb{T}$-valued quadratic form on $\pi_{2}$.

\section{$\S 8.2$. Outline of the general construction}

By now, the reader may have imagined the inductive procedure for constructing the $m$-algebra $R_{m}(X)$. The underlying vector space, or 0-algebra, comprises the (finitely supported) functions on the $m$-truncated homotopy

$$
\coprod_{x \in \pi_{0} X} \pi_{m}(X, x) \times \cdots \times \pi_{1}(X, x) .
$$

As a 1-algebra, we see the $\mathbb{C}\left[\pi_{m}\right]$-valued functions on the union of the $\left(\pi_{m-1} \times \cdots \times \pi_{1}\right)$, with point-wise multiplication. The full $m$-algebra structure can be described recursively. First, as an $(m-1)$-algebra,

$$
R_{m}(X)=\bigoplus_{x \in \pi_{0} X} R_{m-1}\left(\Omega_{x} X\right)
$$

Now, the loop spaces $\Omega_{x} X$ carry a multiplication, and the induced multiplication on each $R_{m-1}$ allows its promotion to an $m$-algebra. Finally, $R_{m}$ is the direct sum of the resulting $m$-algebras.

Remark 8.4. Let us go one step further in unraveling this description: for each base-point $x$, $\pi_{1}(X, x)$ acts on $B^{2} \Omega_{x}^{2} X$; this defines an action on the $m$-algebra $R_{m}\left(B^{2} \Omega_{x}^{2} X\right)$, and we have $R_{m}\left(X_{x}\right):=\pi_{1}(X, x) \ltimes R_{m}\left(B^{2} \Omega_{x}^{2} X\right)$.

This procedure has the advantage of producing finite-dimensional objects whenever the homotopy groups of $X$ are finite, but the choice of base-points in the induction step breaks hopes of functoriality. (This is similar to the construction of a minimal model for $X$ from its Postnikov tower in rational homotopy theory.) So our notation $R_{m}(X)$ is somewhat abusive: we produce something

\footnotetext{
${ }^{20}$ An alternative view: $k$ classifies an extension of $\pi_{1}$ by $B \pi_{2}$, which twists the obvious crossed product tensor structure on $\pi_{1} \ltimes \operatorname{Vect}\left(\pi_{2}^{*}\right)$.
} 
akin to the group ring of the based loop space $\Omega X$. A remedy would be to cross with the Poincaré groupoids, instead of $\pi_{1}$. This would produce a Morita equivalent algebra; but even with a finite model for a higher groupoid, there is then little hope of a finite-dimensional answer.

\section{$\S 8.3$. Twisting by cohomology classes in $\mathbb{T}$}

The construction of the $m$-algebra (and in fact of the entire $n=m+1$-dimensional TQFT) can be twisted by a class $\tau \in H^{n}(X ; \mathbb{T})$ of our space $X$. This gives a projective co-cycle for actions of $\Omega X$ on $(m-1)$-algebras, and the twisted group ring can now again be defined as the $m$-algebra with the 'same' representation category. Some examples: for $m=-1$, the points are now weighted by their phase in $H^{0}(X ; \mathbb{T})$ before counting. For $m=0$, we get a non-trivial action of $\pi_{1}$ on $\mathbb{C}$ for each component of $X$, and only the invariant lines are summed up to produce the vector space. For $m=1$, the class gives a central extension of each $\pi_{1}$, which we quantize to the sum of the corresponding twisted group algebras. For $m=2$ and connected $X, H^{3}(X ; \mathbb{T})$ classifies the crossed homomorphisms $\pi_{1} \rightarrow \pi_{2}^{*}$, and $\tau$ twists the action of $\pi_{1}$ on $\operatorname{Vect}\left(\pi_{2}^{*}\right)$, resulting in a different crossed product $\pi_{1} \ltimes_{\tau, k} \mathbb{C}\left[\pi_{2}\right]$ [2]. Finally, for pure gerbes $K(\pi, 2)$, a 4-class $\tau$ defines a quadratic map $\pi \rightarrow \mathbb{T}$, which defines a braided tensor structure on Vect $[\pi]$. The categories $\operatorname{Sky}^{\tau}[\mathfrak{t}], \operatorname{Sky}^{\tau}[\hat{F}]$ from $\$ 7$ are of this form.

The construction of a general twisted groupoid $m$-algebra follows the inductive procedure sketched earlier. Note that, in the absence of twistings, $R_{m}(X)=R_{m}(X)^{o p}$; this is related to the fact that the associated TQFT does not require orientations.

\section{$\S 8.4$. Construction of the quantization map $\mathrm{Sum}_{n}$}

The category $F H$ of spaces with finitely many, finite homotopy groups has disjoint unions, products and fiber products (homotopy fiber products). As in $₫ 3$, define the $n$-category $F H_{n}$ of correspondences in $F H$ (truncated at level $n$ ). We will enhance the assignment $X \mapsto R_{n-1}(X)$ into a symmetric tensor 21 functor

$$
\operatorname{Sum}_{n}: F H_{n} \rightarrow \operatorname{Alg}[n-1]
$$

into the $n$-category ${ }^{22} \mathrm{Alg}[n-1]$ of $(n-1)$-algebras. Having fixed an $X \in \mathrm{Ob}(F H)$, we pre-compose with the mapping space functor $I: \operatorname{Bord}_{n} \rightarrow F H_{n}$ to produce the 'TQFT with target $X$ ', a theory with values in $\operatorname{Alg}[n-1]$.

Remark 8.6. There is a similar functor $F^{\tau}$ on the category $F H_{n}^{\tau}$, whose objects are spaces with an $n$-co-cycle $\tau$ valued in $\mathbb{T}$, 1-morphisms are correspondences equipped with a homotopy between pulled-back cocycles, and so forth. This leads to the twisted TQFTs for oriented manifolds; we will not spell out its details here.

Whereas on objects $\operatorname{Sum}_{n}(X)=R_{n-1}(X)$, the formula for morphisms is increasingly complex and we pause for a moment to explain why. If $R_{n-1}$ was a contravariant functor with "good" tensor properties, we would just apply it to morphisms of all levels. The reader might think of

\footnotetext{
${ }^{21} \mathrm{Sum}_{n}$ will take disjoint unions to direct sums and fiber products to tensor products.

${ }^{22}$ This is a particular choice of ' $\mathcal{C}$ ' in $\$ 3$
} 
the algebra of co-chains on a space, which converts correspondences to bi-modules. (As a technical aside, this does not always have the required tensor properties, but a closely related functor does: the co-algebra of chains, with co-tensor products.) However, our $R_{n-1}$ is the Koszul dual functor of chains on the based loop space. We thus use Koszul duality as a recipe to define $\mathrm{Sum}_{n}$. The general rule is that a correspondence at level $k$, given by a $k$-storied diagram of spaces, gets sent to the colimit of the same diagram of group rings.

We illustrate this for 1- and 2-morphisms. We may assume connectivity of our spaces (separate components are handled separately). For a correspondence $c: C \rightarrow X \times Y$, call $F$ the homotopy fiber of $c$. It may be viewed as the anti-diagonal quotient $(\Omega X \times \Omega Y) / \Omega C$; the obvious action of $\Omega X \times \Omega Y$ on this last space is the structural one on $F$. To $C$, we assign the $\left(R_{n-1}(X), R_{n-1}(Y)\right)$ bi-module

$$
\operatorname{Sum}_{n}(C):=R_{n-1}(X) \underset{R_{n-1}(C)}{\bigotimes} R_{n-1}(Y) \cong R_{n-2}(F)
$$

This is an $(n-2)$-algebra, as the top multiplication layer has been used up to build the tensor product. The geometric $\Omega X \times \Omega Y$ actions on $F$ induce the bi-module structure on $R_{n-2}(F)$

Now let $D \rightarrow C \times C^{\prime}$ be a correspondence of correspondences; the compositions $D \rightarrow X \times Y$ must agree. With $H$ denoting the homotopy fiber of the last map, there is induced a map $h: H \rightarrow F \times F^{\prime}$ with compatible $\Omega X \times \Omega Y$ action. The homotopy fiber $G$ of $h$ carries $\Omega F \times \Omega F^{\prime}$ actions and intertwining $\Omega X \times \Omega Y$ actions; in fact,

$$
G \sim \frac{\Omega F \times \Omega F^{\prime}}{\Omega H} \sim \frac{\Omega^{2} X \times \Omega^{2} Y}{\Omega^{2} C \underset{\Omega^{2} D}{\times \Omega^{2} C^{\prime}}}
$$

with the left $\times$ right action of $\Omega^{2} C \times \Omega^{2} C^{\prime}$ on each factor in the second numerator; we have just enough commutativity to mod out by the anti-diagonal $\Omega^{2} D$. The desired multi-module is

$$
\operatorname{Sum}_{n}(D):=R_{n-3}(G)=R_{n-2}(F) \underset{R_{n-2}(H)}{\bigotimes} R_{n-2}\left(F^{\prime}\right)=R_{n-2}(X) \underset{R_{n-2}(C) \underset{R_{n-2}(D)}{\otimes} R_{n-2}\left(C^{\prime}\right)}{\bigotimes} R_{n-2}(Y) ;
$$

in the first presentation, tensoring in the $(n-2)$-multiplications ensures that we stay in the realm of " $\left(R_{n-1}(X), R_{n-1}(Y)\right)$ bi-algebra $\left(R_{n-2}(F), R_{(n-2)}\left(F^{\prime}\right)\right)$-bi-algebras", but uses up the top two level products on $\operatorname{Sum}_{n}(D)$ and leaves an $(n-3)$-algebra. In the second presentation, we view $D$ as a $\left(C, C^{\prime}\right)$-correspondence in a lower-dimensional field theory and tensor over the $(n-2)$-algebra $\operatorname{Sum}_{n-1}(D)$, acting left $\times$ right on each factor.

The fun continues, but we will stop.

Remark 8.8. Recall the finite group $F$ from $\$ 5.1$. Consider the gerbe $B^{2} F$, equipped with the class $\tau \in H^{4}\left(B^{2} F ; \mathbb{T}\right)$ described in Lemma 5.17. The space $C$ of maps from a closed 4-manifold $X$ to $B^{2} F$ can be viewed as a top-level correspondence from the point to itself, and (8.2) is the Gauss sum computed in $\$ 5.2$. 


\section{The quantum theories}

The braided tensor categories $\operatorname{Sky}^{\tau}[F], \operatorname{Sky}^{\tau}\left[\mathfrak{t}^{(n)}\right]$ and $\operatorname{Sky}^{\tau}[\mathfrak{t}]$ generate 4-dimensional TQFTs with target space the corresponding ( $\tau$-twisted) gerbes. Their 4-manifold invariants were computed as a path integral in $\$ 5.2$, we have seen their agreement with the numbers provided by the general quantization procedure. The invariants for $\mathfrak{t}$ need to be renormalized by a power of the volume of $\mathfrak{t}$; this is explained by the increasing number of points in its finite approximation $\mathfrak{t}^{(n)}$. We discuss these theories further in $\$ 9.1$.

\section{$\S 9.1$. The quantum gerbe theories on $F, \mathfrak{t}$ and $\mathfrak{t}^{(n)}$}

We now describe the theories $\mathscr{A}_{S}$ on closed manifolds of dimension below $4 ; \mathscr{A}_{\mathbf{t}}$ can be justified either as the limit of $\mathscr{A}_{\mathfrak{t}^{(n)}}$ 's, or in its own right by judicious use of the word 'continuous.' The Lie algebra theory also has a few interesting variations, which tie in with positive energy representations of $L \mathfrak{t}$ and their fusion.

The point. We already know the braided categories $\operatorname{Sky}^{\tau}[\hat{F}], \operatorname{Sky}^{\tau}[\mathfrak{t}]$, and $\operatorname{Sky}^{\tau}\left[\mathfrak{t}^{(n)}\right]$ assigned by $\mathscr{A}$ to a positively oriented point; $\mathscr{A}$ sends the negative point to the opposite object, the category with opposite braiding. Recall that, in all cases, the twisting $\tau \in H^{4}(-; \mathbb{T})$ which defines the braided structure is given by a non-degenerate quadratic map $q$ to $\mathbb{T}$ (see (5.18), (5.22), \$6.1).

The circle. Here, $\mathscr{A}_{F}$ assigns the 2-algebra quantization of the groupoid $L B^{2} F=B F \times B^{2} F$, with twisting $\Omega \tau \in H^{3}\left(L B^{2} F ; \mathbb{T}\right)$ transgressed from $\tau$; this class $\Omega \tau$ represents the bihomomorphism $b: F \times F \rightarrow \mathbb{T}$ derived from $q$. According to $\S 8.3$, the quantization is the crossed product $R:=$ $F \ltimes \Omega \tau \mathbb{C}[F][2]$ with action twisted by the transgression of $\tau$. We have several pictures for this.

(1) The tensor category of $R$-modules is $F \ltimes_{b} \operatorname{Vect}\left(F^{*}\right)$, after identifying $\mathbb{C}[F]$-modules with vector bundles on $F^{*}$. The group $F$ acts on $F^{*}$ by translation, via $b$. Note that this is equivalent to the matrix algebra $M_{F^{*} \times F^{*}}$ (Vect) on $\operatorname{Vect}\left(F^{*}\right)$ !

(2) $R$-modules in algebras are $\mathbb{C}[F]$-algebras with an action of the group $F$ by automorphisms, which twisted commutes with the central $\mathbb{C}[F]: a_{f}\left(f^{\prime}\right)=f^{\prime} \cdot b\left(f, f^{\prime}\right)$ for $f, f^{\prime} \in F$, with $a_{f}$ denoting the automorphism and $f^{\prime} \in F$ embedded in the central $\mathbb{C}[F]$.

(3) Related to this is the 2-category of $R$-linear categories: these are $\mathbb{C}$-linear categories with a projective action of $F \times B F$ with co-cycle $b$. This is an action of $F$ by linear functors plus a second action of $F$ by automorphisms of the identity functor (central automorphisms of all objects), which must be related by $a_{f}\left(f_{x}^{\prime}\right)=f_{a_{f}(x)}^{\prime} \cdot b\left(f, f^{\prime}\right)$, for each object $x$.

(4) The same 2-category has a different presentation which emphasizes its loopy nature: the 2category $\mathscr{B} r^{\tau}(F)$ of (fully) braided bi-module categories over the braided category $\mathrm{Sky}^{\tau}[F]$. The full braiding gives the $B F$-action. We will see that its counterpart $\mathscr{B} r^{\tau}(\mathfrak{t})$ relates nicely to positive energy representations of $L \mathfrak{t}$.

The algebra $\mathbb{C}[F]$ is a module object over $R$ : this is because the linear category $\operatorname{Rep}(F)$ of $\mathbb{C}[F]$-modules is naturally a module over the tensor category $F \ltimes_{b} \operatorname{Vect}\left(F^{*}\right)$ in (i); more precisely, it is the standard simple module of $M_{F^{*} \times F^{*}}$ (Vect). This implies the following.

Proposition 9.1. For non-degenerate $q, R$ is Morita equivalent to $\mathbb{C}[2]$, with bi-module $\mathbb{C}[F]$. 
Closed Surfaces. The space of maps from a closed surface $\Sigma$ to $B^{2} F$ factors as $B^{2} F \times H^{1}(\Sigma ; F) \times$ $F$. The transgression $\tau_{\Sigma}$ of the twisting co-cycle has two components: one of them defines the Heisenberg central extension of $H^{1}(\Sigma ; F)$ constructed from $q$ and the Poincaré duality pairing, the other, on $B^{2} F$, gives the character $f \mapsto b\left(f, f_{0}\right)$, in the component labeled by $f_{0}$. Quantization produces the group ring, the Weyl algebra $W_{\tau}\left(H^{1}(\Sigma ; F)\right)$ for the component $f_{0}=0$, and kills the other components.

At this stage, invertibility of the theory has become obvious, because we get a matrix algebra. Despite this, the theory is not trivial on surfaces. Indeed, while the mapping class group of $\Sigma$ acts by automorphisms on the Weyl algebra, this conceals a central extension by eighth roots of unity, given (when $\mathfrak{t}$ has rank one) by the reduction mod 8 of the Maslov index. This appears when attempting to lift the action on Lagrangian subspaces in $H^{1}$ to the associated Schrödinger representations [P, $\S \mathrm{I} .4]$.

Remark 9.2. Even when this extension is trivial, such as for spin surfaces, two trivializations may differ by a (half-integral) power of the determinant line. This stems from an invertible 3-dimensional theory, and the usual framing anomaly in Chern-Simons theory can be concealed therein.

3-folds with boundary. A bounding 3-manifold $M$ gives a Lagrangian subspace in $H_{1}(\Sigma)$; we can then form the Schrödinger representation $\mathbf{S}$ of $W(\partial M)[\mathrm{P}]$. This is the morphism $\mathscr{A}_{F}(M)$ from $\mathbb{C}$ to the Weyl algebra. Gluing two such manifolds into a closed one produces the line $\operatorname{Hom}_{W(\partial M)}\left(\mathbf{S}_{-}, \mathbf{S}_{+}\right)$. This line has a preferred trivialization on any oriented 3-manifolds, but also carries a natural action of the group $\mathbb{Z}$ of global frame changes.

\section{$\S 9.2$. Chern-Simons theory}

Consider the 3-dimensional gauge theory $Z_{(n)}$ with finite group $T_{(n F)}$ and twisting class $\tau \in$ $H^{3}\left(B T_{(n F)} ; \mathbb{T}\right)$. Such theories were described in $\$ 4.2$. From the perspective of Theorem 2.9 , the theory $Z_{n}$ is generated by the tensor category $\operatorname{Sky}^{\tau}\left[T_{(n F)}\right]$. Similarly, we should view $\mathrm{Sky}^{\tau}[T]$ as the generating tensor category for a theory $Z$, which is an $L^{2}$ version of Chern-Simons theory with gauge group $T$. For example, the vector space associated to a closed surface is the space of $L^{2}$ sections of the Theta-line bundle $\Theta(\tau)$ on the $T$-Jacobian $J_{T}$ of flat bundles (whereas the usual, holomorphic Chern-Simons theory would supply the holomorphic sections). It is easiest to justify the relation of $\mathrm{Sky}^{\tau}[T]$ to $L^{2}$ gauge theory by using the finite approximations $Z_{(n)}$ : sections of $\Theta(\tau)$ on $T_{(n F)}$-bundles approximate $L^{2}\left(J_{T}\right)$ as $n \rightarrow \infty$. We will see in $\S 9.1$ that this goes for 1- and 3-dimensional outputs as well. (The limit must be regularized, so that, for instance, summation over torsion points becomes integration in the limit. $L^{2}$-Chern-Simons itself requires regularization: vectors of bounding 3-manifolds are $\delta$-sections on the respective Lagrangians in $J_{T}$, and the 3-manifold invariant is only 'obviously' finite for for rational homology spheres.)

One picture of a 3-dimensional TQFT, as a stand-alone theory, is as an endomorphism of the trivial 4-dimensional theory, with the top level truncated 24 If, however, a $3 D$ theory is a module over another, non-trivial $4 d$ TQFT $\mathscr{A}$-meaning that the algebras arising various dimensions is

\footnotetext{
${ }^{23}$ In 2-algebra language, $\mathrm{Sky}^{\tau}\left[T_{(n F)}\right]$ is the tensor category of modules for the algebra of functions on $T_{(n F)}$, with $\tau$-twisted Hopf 2-algebra structure.

${ }^{24}$ Without truncation, all morphisms between TQFTs are isomorphisms. At any rate, a $3 D$ TQFT does not supply anything in dimension 4 .
} 
are module objects over their $4 d$ counterparts, in a consistent way - then it can be viewed as a truncated morphism $1 \rightarrow \mathscr{A}$. If $\mathscr{A}$ is invertible, this is the notion of an 'anomalous field theory' with anomaly $\mathscr{A}$. Similarly, a bi-module for theories $\left(\mathscr{A}_{\mathfrak{t}}, \mathscr{A}_{F}\right)$ yields a truncated morphism $\mathscr{A}_{F} \rightarrow \mathscr{A}_{\mathrm{t}}$. Of course, concerning $Z$, we have the gerbe theories for $F$ and $\mathfrak{t}$ in mind. The following summarizes our results.

Theorem 9.3. (i) The $L^{2}$-Chern-Simons theory $Z$ generated by $\mathrm{Sky}^{\tau}[T]$ gives an isomorphism $\mathscr{A}_{F} \rightarrow \mathscr{A}_{\mathrm{t}}$ of oriented $4 d$ theories.

(ii) The finite gauge theory $Z_{(n)}$ generated by $\mathrm{Sky}^{\tau}\left[T_{(n F)}\right]$ gives an isomorphism $\mathscr{A}_{F} \rightarrow \mathscr{A}_{\mathfrak{t}^{(n)}}$ of oriented $4 d$ theories.

(iii) $Z$ gives a truncated morphism $1 \rightarrow \mathscr{A}_{\mathfrak{t}}$ of oriented $4 d$ theories.

(iv) A Morita equivalence Vect $\sim \mathrm{Sky}^{\tau}[\mathfrak{t}]$ induces an isomorphism $\mathscr{A}_{\mathfrak{t}} \rightarrow 1$; after a composition with such an isomorphism, $Z$ becomes isomorphic to $3 d$ (holomorphic) Chern-Simons theory.

(v) $Z_{(n)}$ gives a truncated morphism $1 \rightarrow \mathscr{A}_{\mathfrak{t}^{(n)}}$ of oriented $4 d$ theories. A Morita equivalence Vect $\sim$ $\mathrm{Sky}^{\tau}\left[\mathfrak{t}^{(n)}\right]$ induces an isomorphism $\mathscr{A}_{\mathfrak{t}^{(n)}} \rightarrow 1$; after a composition with such an isomorphism, $Z_{(n)}$ becomes isomorphic to $3 d$ (holomorphic) Chern-Simons theory.

Remark 9.4. Recall from $\S 6.3$ that a Morita isomorphism as in (iii) and (v) can be found whenever 8 divides the signature of $\tau$, or anytime we work with graded objects and spin manifolds. Items (ii) and (v) are perhaps not of much interest, but in the $n \rightarrow \infty$ limit they serve to justify rigorously the claims (i), (iii) and (iv); without that, we would need to delve into topological categories and their continuous centers. The truncated morphism $Z$ in (iii) is (holomorphic) Chern-Simons as an anomalous theory.

The theorem is really a corollary of Proposition 6.5 or at least it would be so, if we supplied the information needed to make the braided tensor categories into fully dualizable objects with $S O(4)$ actions in the world of braided tensor categories 25 We shall not do this; instead let us explain what the theories assign in each dimension. In particular, we will recover the usual modular tensor category [St for holomorphic Chern-Simons for $T$ as the relative Drinfeld center of $\mathrm{Sky}^{\tau}[T]$ over $\mathrm{Sky}^{\tau}[\mathfrak{t}]$.

\section{$\S 9.3$. Chern-Simons as an anomalous theory}

We condense the relations between our theories on closed manifolds $X$ in the following table, in which the third column gives a Morita isomorphism between the second and fourth columns. We have written $Z(X)$ for the $L^{2}$ Chern-Simons invariant of a 3-manifold, a renormalized count of the flat $T$-bundles on $X\left[\mathrm{Ma}\right.$, while $\mathscr{A}_{F, \mathfrak{t}}(X)$ refers to the 4-manifold invariants computed in $\S 5.2$.

\footnotetext{
${ }^{25}$ Something is missing, as can be seen from the invariant computation in $\$ 5.2$ where an Euler characteristiccoupled parameter must be supplied.
} 


\begin{tabular}{cccc}
\hline $\operatorname{dim} X$ & $\mathscr{A}_{\mathfrak{t}}(X)$ & $Z(X)$ & $\mathscr{A}_{F}(X)$ \\
\hline 0 & $\operatorname{Sky}^{\tau}[\mathfrak{t}]$ & $\operatorname{Sky}^{\tau}[T]$ & $\operatorname{Sky}^{\tau}[\hat{F}]$ \\
1 & $\mathfrak{t} \ltimes_{b} \operatorname{Sky}_{\left.\mathfrak{t}^{*}\right]}$ & $\operatorname{Sky}^{\tau}[C]$ & $\left.F \ltimes_{b} \operatorname{Sky}^{*}\left[F^{*}\right]\right)$ \\
2 & $W_{\tau}\left(H^{1}(X ; \mathfrak{t})\right)$ & $L^{2}\left(J_{T}(X) ; \Theta(\tau)\right)$ & $W_{\tau}\left(H^{1}(X ; F)\right)$ \\
3 & $\mathbb{C}$ & $Z(X)$ & $\mathbb{C}$ \\
4 & $\mathscr{A}_{\mathfrak{t}}(X)$ & - & $\mathscr{A}_{F}(X)$ \\
\hline
\end{tabular}

Theories on closed manifolds

There is a matching equivalence with the finite theories for $T_{(n)}, \mathfrak{t}^{(n)}$ :

\begin{tabular}{cccc}
\hline $\operatorname{dim} X$ & $\mathscr{A}_{\mathfrak{t}^{(n)}}(X)$ & $Z_{(n)}(X)$ & $\mathscr{A}_{F}(X)$ \\
\hline 0 & $\operatorname{Sky}^{\tau}\left[\mathfrak{t}^{(n)}\right]$ & $\operatorname{Sky}^{\tau}\left[T_{(n F)}\right]$ & $\operatorname{Sky}^{\tau}[\hat{F}]$ \\
1 & $\mathfrak{t}^{(n)} \ltimes_{b} \mathrm{Sky}\left[\mathfrak{t}^{*(n)}\right]$ & $\operatorname{Sky}^{\tau}\left[C^{(n)}\right]$ & $\left.\left.F \ltimes_{b} \mathrm{Sky}^{(n)}\right]\right)$ \\
2 & $W_{\tau}\left(H^{1}\left(X ; \mathfrak{t}^{(n)}\right)\right)$ & $L^{2}\left(J_{T_{(n F)}}(X) ; \Theta(\tau)\right)$ & $W_{\tau}\left(H^{1}(X ; F)\right)$ \\
3 & $\mathbb{C}$ & $Z_{(n)}(X)$ & $\mathbb{C}$ \\
4 & $\mathscr{A}_{\mathfrak{t}^{(n)}}(X)$ & - & $\mathscr{A}_{F}(X)$ \\
\hline
\end{tabular}

Finite theories on closed manifolds

Experts may have recognized in the right column the double of Chern-Simons theory for the torus $T$ at level $\tau$. This is explained as follows.

The second and third columns in each table give our advertised description of (holomorphic) Chern-Simons theory as an anomalous theory. We claim that $Z$ is 'finite as a module over $\mathscr{A}_{\mathfrak{t}}$, with holomorphic Chern-Simons theory as a basis' (and similarly for $Z_{(n)}$ and $\mathscr{A}_{\mathbf{t}^{(n)}}$ ). The most obvious instance is that of the vector space

$$
Z(\Sigma)=L^{2}\left(J_{T}(\Sigma) ; \Theta(\tau)\right)
$$

for a closed surface $\Sigma$. The theory of Theta-functions tells us that, after a choice of complex structure, (9.5) factors into holomorphic and anti-holomorphic sectors; the former is the space of holomorphic Theta-functions, an irreducible representation of the Heisenberg group on $\left.H^{(} \Sigma ; F\right)$, and the latter the anti-holomorphic Fock representation of the Weyl algebra $W\left(H^{1}(\Sigma ; \mathfrak{t})\right)$.

One dimension down, the center $\mathrm{Sky}^{\tau}[C]$ of $\mathrm{Sky}^{\tau}[T]$, plays the role of the modular tensor category for $L^{2}$ Chern-Simons. The braided structure on $\operatorname{Sky}^{\tau}[C]$ makes this into a braided bi-module over $\mathrm{Sky}^{\tau}[\mathfrak{t}]$, thus a $\mathfrak{t} \ltimes_{b} \operatorname{Sky}\left[\mathfrak{t}^{*}\right]$-module. It is free with basis $\mathrm{Sky}^{\tau}[\hat{F}]$, in the sense that it converts into the latter, after the Morita equivalence of $\mathfrak{t} \ltimes_{b} \operatorname{Sky}^{\tau}\left[\mathfrak{t}^{*}\right]$ with 1 defined by Sky $\left[\mathfrak{t}^{*}\right]$. Such a Morita equivalence can be induced from a trivialization of $\mathscr{A}_{\mathbf{t}}$ by means of $\mathrm{Sky}^{\tau^{\prime}}[T]$ at a level $\tau^{\prime}$ giving a trivial group $F^{\prime}, 26$ for indeed, $\mathrm{Sky}^{\tau^{\prime}}\left[C^{\prime}\right]$ is then just $\mathrm{Sky}\left[\mathfrak{t}^{*}\right]$, as a braided bi-module. (The change

${ }^{26}$ Using graded vector spaces, see $\S 6.3$. 
to another level of the same signature can be accommodated by scaling the Lie algebra.) This is also related to the computation of the relative center of $\operatorname{Sky}^{\tau}[T]$ over the braided category $\operatorname{Sky}^{\tau}[\mathfrak{t}]$.

In dimension 0, our data for Chern-Simons theory is new.

\section{$\S 9.4$. Surfaces with boundary in another model for $\mathfrak{t}$-gerbe theory}

In this model $\operatorname{Rep}(L \mathfrak{t})$ for $\mathrm{Sky}^{\tau}[\mathfrak{t}]$, closely related to loop groups, the objects are semi-simple, projective, positive energy modules of the smooth loop Lie algebra $L \mathfrak{t}$, with projective co-cycle

$$
(\xi, \eta) \mapsto \oint\langle\xi, d \eta\rangle
$$

These representations are invariant under diffeomorphisms of the circle; non-invariantly, they factor into a semi-simple representation of $\mathfrak{t}$ and the Fock representation of $L \mathfrak{t} / \mathfrak{t}$, the latter being a Morita factor in the equivalence.

The fusion of two representations is defined using a pair of pants $P$ : the Weyl algebra $W_{\tau}(P)$ of the pair of pants (see below) accepts maps from three commuting copies of the Weyl algebra $W_{\tau}(L \mathfrak{t})$ of $L \mathfrak{t}$. The fusion of two boundary representations is the induced module from their tensor product, as a $W_{\tau}(P)$-module, to the the third boundary. Fusion gives a braided tensor structure on $\operatorname{Rep}(L \mathfrak{t})$, which makes it equivalent to $\operatorname{Sky}^{\tau}[\mathfrak{t}] . W_{\tau}(L \mathfrak{t})$ itself is the underlying 3-algebra, when equipped with the fusion product via the braided tri-module $W_{\tau}(P)$.

Associated to a surface $\Sigma$ with boundary is the symplectic vector space $S_{\Sigma}:=\Omega^{1}(\Sigma) / d \Omega^{0}(\Sigma, \partial \Sigma)$ of closed forms modulo differentials of functions vanishing on $\partial \Sigma$; the symplectic form is $\int_{\Sigma} \varphi \wedge \psi$. The Weyl algebra $W_{\tau}\left(S_{\Sigma} \otimes \mathfrak{t}\right)$ is a braided bi-algebra for the $W_{\tau}(L \mathfrak{t})$ 's at each boundary circle. This promotes $W_{\tau}(\Sigma)$ to an object of $\mathscr{B} r^{\tau}$, the 2-category (equivalent to that) of $\mathfrak{t} \ltimes_{b}$ Sky[ $\left.\mathfrak{t}^{*}\right]$-modules, and the thus promoted $W_{\tau}(\Sigma)$ is $\mathscr{A}_{\mathfrak{t}}(\Sigma)$.

A model $S_{\Sigma}^{\prime}$ for $S_{\Sigma}$ that removes the Morita factors $\operatorname{Fock}(L \mathfrak{t} / \mathfrak{t})$ from $W$ uses only differentials whose restriction to each boundary circle is constant (in a fixed parameterization). We lose the connection to loop groups, but this is convenient for describing $Z(\Sigma)$ (without having to Moritamodify $C$ ). Now, $Z(\Sigma)$ is a functor between products of copies of $\mathrm{Sky}^{\tau}[C]$, one for each boundary circle: in effect, a vector bundle over a product $C^{n}$. The moduli space $J_{T}(\Sigma, \partial \Sigma)$ of flat $T$-bundles on $\Sigma$ equipped with constant connections on $\partial \Sigma$ projects to $\mathfrak{t}^{n}$ by the boundary holonomies; call this map p. $Z(\Sigma)$ is the bundle over $\mathfrak{t}^{n}$ of fiber-wise $L^{2}$ sections of $\Theta(\tau)$ along $p$; it is naturally a

finite module over $W_{\tau}\left(S^{\prime} \otimes \mathfrak{t}\right)$ under its translation of the Jacobian. The $\hat{F}$-components of $Z(\Sigma)$ are determined by the weight space decomposition under the actions of $F$ at the boundaries.

\section{References}

[A] M. Atiyah, Topological quantum field theories, Inst. Hautes Études Sci. Publ. Math. (1988), no. 68, 175-186 (1989).

[AS] M. Atiyah, G. Segal, Twisted K-theory, Ukr. Mat. Visn. 1 (2004), no. 3, 287-330.

[BD] J. C. Baez, J. Dolan, Higher-dimensional algebra and topological quantum field theory, J. Math. Phys. 36 (1995), no. 11, 6073-6105. 
[BK] B. Bakalov, A. Kirillov, Jr., Lectures on tensor categories and modular functors, University Lecture Series, vol. 21, American Mathematical Society, Providence, RI, 2001.

[BW] J. W. Barrett, B. W. Westbury, Spherical categories, Adv. Math., 143 (1999), 357-375, arXiv:hep-th/9310164

[BM] D. Belov, G. W. Moore, Classification of abelian spin Chern-Simons theories, arXiv:hep-th/0505235

[BN] D. Ben-Zvi, D. Nadler, The character theory of a complex group, arXiv:0904.1247.

[B] R. Bott, Lectures on characteristic classes and foliations, Lectures on algebraic and differential topology (Second Latin American School in Math., Mexico City, 1971), Springer, Berlin, 1972, Notes by Lawrence Conlon, with two appendices by J. Stasheff, pp. 1-94. Lecture Notes in Math., Vol. 279.

[Bry] J-L Brylinski, Gerbes on complex reductive Lie groups, arXiv:math/0002158.

[CY] L. Crane, D. Yetter, A categorical construction of 4D topological quantum field theories, Quantum topology, Ser. Knots Everything, vol. 3, World Sci. Publ., River Edge, NJ, 1993, pp. 120-130.

[D] C. Douglas, Two-dimensional algebra and quantum Chern-Simons theory, lecture at Topological Field Theory conference, Northwestern University, May, 2009.

[EL] S. Eilenberg, S. Mac Lane, On the groups $H(\Pi, n)$. II. Methods of computation, Ann. of Math. 60 (1954), 49-139.

[FHT] C. Teleman D. S. Freed, M. J. Hopkins, Loop groups and twisted K-theory I, II, III, arXiv:0711.1906, arXiv:math/0511232, arXiv:math/0312155.

[F1] D. S. Freed, Classical Chern-Simons theory. II, Houston J. Math. 28 (2002), no. 2, 293-310, Special issue for S. S. Chern.

[F2] Higher algebraic structures and quantization, Comm. Math. Phys. 159 (1994), no. 2, 343-398.

[HS] M. J. Hopkins, I. M. Singer, Quadratic functions in geometry, topology, and M-theory, J. Differential Geom. 70 (2005), no. 3, 329-452.

[HM] D. Husemoller, J. Milnor, Symmetric bilinear forms, Springer-Verlag, New York, 1973, Ergebnisse der Mathematik und ihrer Grenzgebiete, Band 73.

[L] J. Lurie, On the classification of topological field theories, available at http://www-math.mit.edu/ lurie/

[Ma] M. Manoliu, Abelian Chern-Simons theory. I. A topological quantum field theory, J. Math. Phys. 39 (1998), no. 1, 170-206. II. A functional integral approach, J. Math. Phys. 39 (1998), no. 1, 207-217.

[M] M. Müger, From subfactors to categories and topology. II. The quantum double of tensor categories and subfactors, J. Pure Appl. Algebra 180 (2003), no. 1-2, 159-219.

$[\mathrm{P}] \quad$ A. Polishchuk, Abelian varieties, theta functions and the Fourier transform, Cambridge Tracts in Mathematics, 153, Cambridge University Press, Cambridge, 2003.

[Q] F. Quinn, Lectures on axiomatic topological quantum field theory, in Geometry and Quantum Field Theory, IAS/Park City Mathematics Series, Volume 1, D. S. Freed and K. K. Uhlenbeck, eds., American Mathematical Society, Providence, RI, 1995, pp. 323-453.

[S] G. B. Segal, Stanford Lectures, Lecture3l.H 